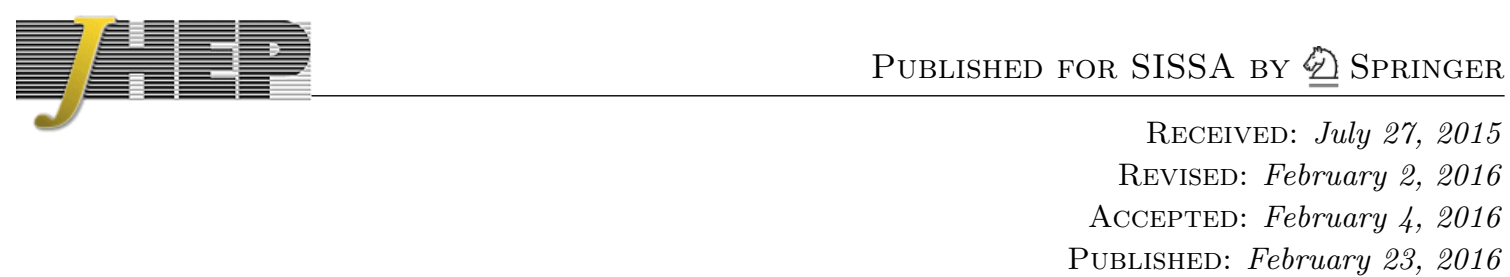

\title{
Quantum entanglement of fermionic local operators
}

\section{Masahiro Nozaki, Tokiro Numasawa and Shunji Matsuura}

Yukawa Institute for Theoretical Physics,

Kyoto University, Kyoto 606-8502, Japan

E-mail: masahiron@uchicago.edu, numasawa@yukawa.kyoto-u.ac.jp,

matsuura@nbi.ku.dk

ABSTRACT: In this paper we study the time evolution of (Rényi) entanglement entropies for locally excited states in four dimensional free massless fermionic field theory. Locally excited states are defined by being acted by various local operators on the ground state. Their excesses are defined by subtracting (Rényi) entanglement entropy for the ground state from those for locally excited states. They finally approach some constant if the subsystem is given by half of the total space. They have spin dependence. They can be interpreted in terms of quasi-particles.

KEywords: Field Theories in Lower Dimensions, Conformal and W Symmetry, Field Theories in Higher Dimensions

ARXIV EPRINT: 1507.04352 


\section{Contents}

1 Introduction $\quad 2$

2 Setup 3

3 Replica trick $\quad 4$

3.1 Locally excited states 4

3.2 Replica method 5

4 Propagator $\quad 6$

$\begin{array}{lll}4.1 & \text { Computation of propagator } & 7\end{array}$

4.2 Analytic continuation to real time 9

$\begin{array}{lll}4.3 & \text { Dominant propagators } & 10\end{array}$

4.3.1 Propagators in canonical frame in the Cartesian coordinate 11

$5 \Delta S_{A}^{(n)}$ for various local operators $\quad 13$

$5.1 \Delta S_{A}^{(n)}$ for $\psi_{a}^{\prime} \quad 13$

5.1.1 The excess of Rényi entanglement entropies 13

$\begin{array}{lll}5.1 .2 & \text { Reduced density matrix } & 14\end{array}$

$\begin{array}{lll}5.2 \Delta S_{A}^{(n)} \text { for } \bar{\psi}^{\prime} \psi^{\prime} & 15\end{array}$

$\begin{array}{lll}\text { 5.2.1 The excess of (Rényi) entanglement entropy } & 15\end{array}$

$\begin{array}{lll}5.2 .2 & \text { Reduced density matrix } & 16\end{array}$

$\begin{array}{lll}5.3 \Delta S_{A}^{(n)} \text { for } \psi^{\prime \dagger} \psi^{\prime} & 17\end{array}$

$\begin{array}{lll}\text { 5.3.1 The excess of (Rényi) entanglement entropy } & 17\end{array}$

$\begin{array}{lll}5.4 & \text { Reduced density matrix } & 18\end{array}$

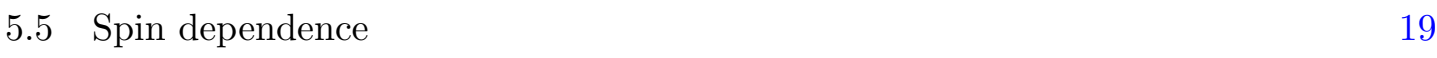

5.5.1 Spin dependence of time evolution 21

6 Quasi-particle interpretation $\quad 22$

6.1 Various examples 23

6.1.1 $\rho_{A}$ for $\psi_{a} \quad 23$

6.1.2 $\rho_{A}$ for $\bar{\psi} \psi \quad 24$

6.1.3 $\rho_{A}$ for $\psi^{\dagger} \psi \quad 25$

$\begin{array}{lll}7 & \text { Conclusions and discussions } & 25\end{array}$ 


\section{Introduction}

Quantum entanglement is an essential idea that distinguishes quantum physics from classical physics. There has been a lot of work done to investigate various subfields of physics from a perspective of quantum entanglement. For instance, the universal properties of quantum entanglement are used to characterize conformal field theories [1-5], as well as topologically ordered phases $[6,7]$. The quantum entanglement also plays an essential role to understand the Hilbert space of gravity [8-13]. While the most commonly used quantities for characterizing states are correlation functions of operators, quantum entanglement focuses more directly on the Hilbert space.

Entanglement entropy is defined as the von Neumann entropy of a reduced density matrix $\rho_{A}$ for a subsystem $A$. It has been observed that the dominant contribution of entanglement entropy in ground states of gapped systems comes from near boundary region of $A$. This leads to the area law of entanglement entropy [14]. For ground states of gapless systems, the contribution of the entanglement from a long distance does not necessarily become subdominant. Indeed, in $2 \mathrm{~d}$ conformal field theories (CFTs), entanglement entropy behaves universally as $\frac{c}{3} \log l$ where $c$ is the central charge and $l$ is the size of the subsystem $A$, suggesting that entanglement of any distance equally contributes to the entropy. Recently some entanglement measures are introduced to describe the more detail structure of the entanglement entropy $[15,16]$.

The above story is only for static (ground) states and much less is understood for the dynamical aspects of the entanglement entropy as well as the entanglement entropy for excited states. There are interesting questions about the dynamics of quantum entanglement, such as how the quantum entanglement is created and propagates, how they distributes in space time. Those are essential for understanding far-equilibrium states and their thermalization [17], how efficiently quantum dynamics can be simulated in classical computers, and where and how the black hole information goes.

In this paper, we study the time evolution of (Rényi) entanglement entropies by being acted by a fermionic local operator on the ground state. First we explain quantum quenches which are a useful protocol to investigate the dynamical aspects of the entanglement. Suppose a system is prepared for a Hamiltonian $H\left(\lambda_{0}\right)$ which has an experimentally controllable parameter $\lambda_{0}$. The state prepared for this system is given by a ground state. Then at a certain time the parameter is shifted from $\lambda_{0}$ to $\lambda_{1}$. The prepared state is no longer a ground state of $H\left(\lambda_{1}\right)$ and the state undergoes a time evolution. This kind of control is indeed possible in experiments such as cold atom systems [18]. When parameters are changed globally, these protocols are called as global quenches [19]. On the other hand, it is called as local quenches if parameters are changed locally [20, 21].

Our protocol is similar to local quenches. In our setup, parameters of Hamiltonian are not changed. Instead of being changed them, a local operator $\mathcal{O}\left(t_{0}, x_{0}\right)$ acts on the ground state at certain time and creates entangled quasi-particle. In CFTs they propagate spherically at the speed of light. If this local operator is inserted outside of $A$, a part of quasi-particles eventually enters into the subsystem $A$ and the rest part stays outside. Since they are entangled, it may create an entanglement between the subsystem $A$ and 
the rest of the system. Indeed this kind of behavior, the increase of the entanglement and the saturation, has been observed in various quantum field theories [22-29]. On the other hand, in holographic field theories the excesses of (Rényi) entanglement entropies do not saturate and keep to increase logarithmically even if time passes sufficiently (at late time) $[25,30,31]$.

The standard method for computing the entanglement entropy for ground states or thermal states in path integral is the replica method: we compute the (Rényi) entanglement entropy $S_{A}^{(n)}=\frac{1}{1-n} \log \operatorname{tr}_{A} \rho_{A}^{n}$ first and then take $n \rightarrow 1$ limit, which gives entanglement entropy $S_{A}=-\operatorname{tr}_{A} \rho_{A} \log \rho_{A}$. Here $\rho_{A}=\operatorname{tr}_{B} \rho$ and we trace out the degrees of freedom outside $A$ (the region $B$ ).

We consider free massless fermionic field theory in 4 dimensional spacetime and take a half space as the subsystem $A$. We generate an excitation by being acted by local operator $\mathcal{O}$ at a distance $l$ away from the entangling surface and time $-t$. The state prepared is given by a locally excited state.

$$
|\Psi\rangle=\mathcal{N O}(-t,-l, \mathbf{x})|0\rangle,
$$

where $\mathbf{x}=\left(x_{2}, x_{3}\right)$. We define the excesses of (Rényi) entanglement entropies $\Delta S_{A}^{(n)}$ by subtracting (Rényi) entanglement entropies for the ground state from those for locally excited states.

They do not change for $t<l$, as expected from the causality. The main difference is the final values of $\Delta S_{A}^{(n)}$ : in the case of free massless fermionic field case, $\Delta S_{A}^{(n)}$ have spin dependence. Their density matrices can depend on the direction of spin because probability with which (anti-)particles are included in $A$ can depend on the direction of spin. In the free massless scalar field theory, of course $\Delta S_{A}^{(n)}$ do not have such a dependence.

This paper is organized as follows.

In section 2, we explain our setup. In section 3, we explain the replica method and the analytic continuation which we perform. In section 4 we derive the Green function in $4 d$ free massless fermionic field theory on the replica space. In section 5 we compute the time evolution of $\Delta S_{A}^{(n)}$. In section 6 we explain the same thing in terms of quasi-particles. In section 7 , we conclude and discuss our results.

\section{Setup}

We study the time evolution of excesses of (Rényi) entanglement entropies for locally excited states which are defined by being acted by various local operators on the ground states in following setup. We consider the 4 dimensional free massless fermionic theory,

$$
S_{\text {fermion }}=-\int d^{4} x \bar{\psi} \gamma^{\mu} \partial_{\mu} \psi
$$

where $\gamma^{\mu}=\left\{\gamma^{t}, \gamma^{1}, \gamma^{2}, \gamma^{3}\right\}$ and $\bar{\psi}=i \psi^{\dagger} \gamma^{t}$.

A local operator $\mathcal{O}$ acts on the ground state as in figure.1. A locally excited state is given by

$$
|\Psi\rangle=\mathcal{N O}(-t,-l, \mathbf{x})|0\rangle,
$$

where $\mathbf{x}=\left(x^{2}, x^{3}\right)$ and $\mathcal{N}$ is a normalization constant. 


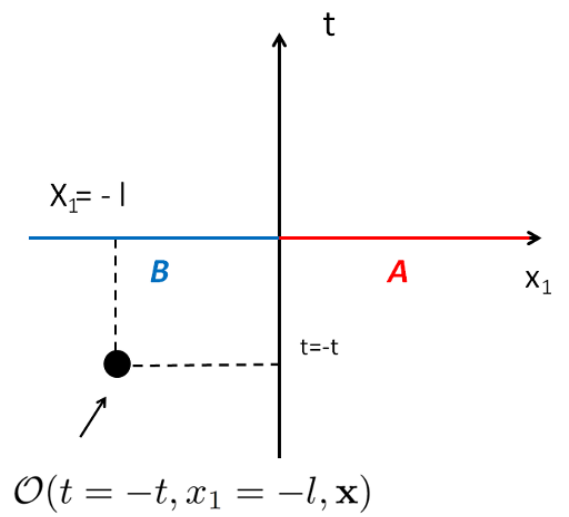

Figure 1. The location of an local operator and the subsystem $A$ in the Minkowski spacetime.

The subsystem $A$ is given by a half of the total space, $x^{1} \geq 0$ as in figure.1. We trace out the degrees of freedom in the complement space $B$ outside $A$ and define a reduced density matrix,

$$
\rho_{A}^{E X}=\operatorname{tr}_{B} \rho^{E X}
$$

where $\rho=|\Psi\rangle\langle\Psi|$.

By using this reduced density matrix, (Rényi) entanglement entropy for locally excited state is defined by

$$
S_{A}^{(n) E X}=\frac{1}{1-n} \log \left[\operatorname{tr}_{A}\left(\rho_{A}^{E X}\right)^{n}\right]
$$

By using the reduced density matrix for the ground state $\rho_{A}^{G}=\operatorname{tr}_{B}|0\rangle\langle 0|$, (Rényi) entanglement entropy for the ground state is defined by

$$
S_{A}^{(n) G}=\frac{1}{1-n} \log \left[\operatorname{tr}_{A}\left(\rho_{A}^{G}\right)^{n}\right] .
$$

We define the excess of (Rényi) entanglement entropy by subtracting $S_{A}^{(n) G}$ from $S_{A}^{(n) E X}$,

$$
\Delta S_{A}^{(n)}=S_{A}^{(n) E X}-S_{A}^{(n) G} .
$$

We will study the time evolution of $\Delta S_{A}^{(n)}$ in following sections.

\section{Replica trick}

\subsection{Locally excited states}

In this section, we explain the replica method for locally excited states. The states we are considering are given as follows:

$$
|\Psi\rangle=\mathcal{N} e^{-i H t} e^{-\epsilon H} \mathcal{O}(-l, \mathbf{x})|0\rangle .
$$

Here $\mathcal{O}(-l, \mathbf{x})$ is a local operator in Shrödinger picture and we introduce the regularization factor $e^{-\epsilon H}$ because the norm of the state $|\Psi\rangle$ is divergent without $e^{-\epsilon H}$ and $|\Psi\rangle$ does not belong to the Hilbert space. This corresponds to smearing the point like excitation. 
The density matrix $\rho$ is given by

$$
\begin{aligned}
\rho & =\mathcal{N}^{2} e^{-i H t} e^{-\epsilon H} \mathcal{O}(-l, \mathbf{x})|0\rangle\langle 0| \mathcal{O}(-l, \mathbf{x})^{\dagger} e^{-\epsilon H} e^{i H t} \\
& =\mathcal{N}^{2} \mathcal{O}\left(\tau_{e},-l, \mathbf{x}\right)|0\rangle\langle 0| \mathcal{O}^{\dagger}\left(\tau_{l},-l, \mathbf{x}\right)
\end{aligned}
$$

Here $\mathcal{O}(\tau,-l, \mathbf{x})$ is a local operator in Heisenberg picture and in the second line we introduce complex times $\tau_{e}=-\epsilon-i t$ and $\tau_{l}=\epsilon-i t$. In the calculation of (Rényi) entanglement entropy, we first treat complex times $\tau_{e}$ and $\tau_{l}$ as if they are real parameters and finally we analytically continue to complex values.

\subsection{Replica method}

Now we explain the replica method for locally excited states. In the path integral formalism, we can express the wave functional for locally excited states is given by

$$
\Psi(\phi(x))=\left(Z_{1}^{E X}\right)^{-\frac{1}{2}}\langle\phi \mid \Psi\rangle=\int_{\phi\left(\tau=-\infty, x^{i}\right)}^{\phi\left(\tau=0, x^{i}\right)=\phi\left(x^{i}\right)} \mathcal{D} \phi \mathcal{O}\left(\tau_{e},-l, \mathbf{x}\right) e^{-S[\phi]},
$$

where $\left(Z_{1}^{E X}\right)^{1 / 2}=\mathcal{N}$. In the same manner, the we can express the bra vector as follows:

$$
\Psi^{*}(\phi(x))=\left(Z_{1}^{E X}\right)^{-\frac{1}{2}}\langle\Psi \mid \phi\rangle=\int_{\phi\left(\tau=0, x^{i}\right)=\phi\left(x^{i}\right)}^{\phi\left(\tau=\infty, x^{i}\right)} \mathcal{D} \phi \mathcal{O}^{\dagger}\left(\tau_{l},-l, \mathbf{x}\right) e^{-S[\phi]} .
$$

Then, the density matrix for total system is given by the path integral on the space which has boundary at $\tau=+0$ and $\tau=-0$ :

$$
\begin{aligned}
{\left[\rho_{\mathrm{tot}}\right]_{\phi \phi^{\prime}} } & =\langle\phi \mid \Psi\rangle\left\langle\Psi \mid \phi^{\prime}\right\rangle \\
& =\left(Z_{1}^{E X}\right)^{-1} \int_{\phi\left(\tau=+0, x^{i}\right)=\phi^{\prime}\left(x^{i}\right), \phi\left(\tau=-0, x^{i}\right)=\phi\left(x^{i}\right)} \mathcal{D} \phi \mathcal{O}^{\dagger}\left(\tau_{l},-l, \mathbf{x}\right) \mathcal{O}\left(\tau_{e},-l, \mathbf{x}\right) e^{-S[\phi]}
\end{aligned}
$$

where $Z_{1}^{E X}$ appears in order to keep $\operatorname{tr} \rho=1$ and it is given by

$$
Z_{1}^{E X}=\mathcal{N}^{2}=\int_{\phi\left(\tau=-\infty, x^{i}\right)}^{\phi\left(\tau=\infty, x^{i}\right)} \mathcal{D} \phi \mathcal{O}^{\dagger}\left(\tau_{l},-l, \mathbf{x}\right) \mathcal{O}\left(\tau_{e},-l, \mathbf{x}\right) e^{-S[\phi]} .
$$

Partial trace corresponds to sawing the region which was traced out, so the reduced density matrix is given by

$$
\begin{aligned}
{\left[\rho_{A}^{E X}\right]_{\phi \phi^{\prime}}=\left(Z_{1}^{E X}\right)^{-1} \int_{\phi\left(\tau=-\infty, x^{i}\right)}^{\phi\left(\tau=\infty, x^{i}\right)} } & \mathcal{D} \phi \mathcal{O}^{\dagger}\left(\tau_{l},-l, \mathbf{x}\right) \mathcal{O}\left(\tau_{e},-l, \mathbf{x}\right) e^{-S[\phi]} \\
& \times \prod_{x \in A} \delta\left(\phi\left(+0, x^{i}\right)-\phi^{\prime}\left(x^{i}\right)\right) \cdot \delta\left(\phi\left(-0, x^{i}\right)-\phi\left(x^{i}\right)\right) .
\end{aligned}
$$

From this we can see that the only difference between the reduced density matrix for ground states and that for locally excited states is the insertion of local operators $\mathcal{O}\left(\tau_{e}\right)$ and $\mathcal{O}\left(\tau_{l}\right)$. We need to insert two local operators in each sheet, so finally we need to insert $2 n$ local 


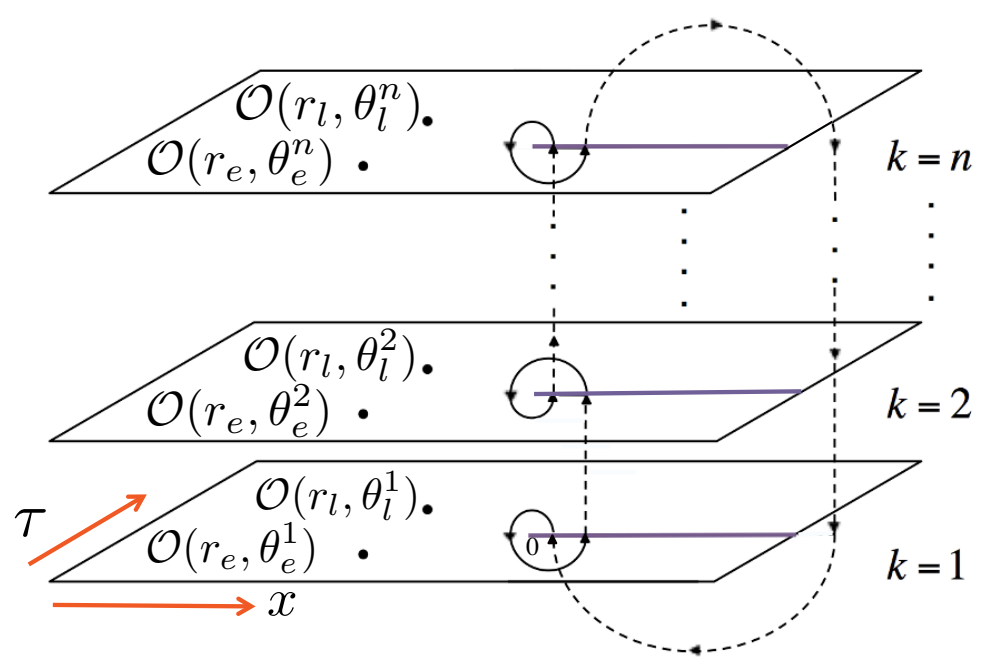

Figure 2. $n$-sheeted manifold with operator insertion.

operators in the $n$-sheeted manifold $\Sigma_{n}$ which is constructed of $n$ flat spaces and it has a conical singularity on the entangling surface as in figure 2 .

Then, the $\operatorname{tr}\left(\rho_{A}^{E X}\right)^{n}$ is given by the partition function with the insertion of $2 n$ local operators:

$$
\operatorname{tr}\left(\rho_{A}^{E X}\right)^{n}=\left(Z_{1}^{E X}\right)^{-n} \int \mathcal{D} \phi \mathcal{O}^{\dagger}\left(r_{l}, \theta_{l}^{n}\right) \mathcal{O}\left(r_{e}, \theta_{e}^{n}\right) \cdots \mathcal{O}^{\dagger}\left(r_{l}, \theta_{l}^{1}\right) \mathcal{O}\left(r_{e}, \theta_{e}^{1}\right) e^{-S[\phi]}
$$

where we introduce the polar coordinate $(r, \theta)$ on $\left(\tau, x_{1}\right)$ plane, the region of $\theta$ is given by $0<\theta<2 \pi n$ and $\theta_{e, l}^{k}=\theta_{e, l}^{1}+2 \pi(k-1)$, see figure 2. Eq. (3.8) is almost the correlation function and the only difference is that right hand side is divided by $\left(Z_{1}^{E X}\right)^{n}$, not by $Z_{n}$ where $Z_{n}$ is the partition function on $n$-sheeted manifold $\Sigma_{n}$ without any operator insertion. If we consider the difference between Rényi entanglement entropy for excited states and that for the ground state, we can find that it is expressed by the correlation function on $n$-sheeted manifold:

$$
\begin{aligned}
\Delta S_{A}^{(n)}= & \frac{1}{1-n}\left(\log \frac{\operatorname{tr}_{A}\left(\rho_{A}^{E X}\right)^{n}}{\left(\operatorname{tr}_{A} \rho_{A}^{E X}\right)^{n}}-\log \frac{\operatorname{tr}_{A}\left(\rho_{A}^{G}\right)^{n}}{\left(\operatorname{tr}_{A} \rho_{A}^{G}\right)^{n}}\right)=\frac{1}{1-n}\left(\log \frac{Z_{n}^{E X}}{Z_{n}}-n \log \frac{Z_{1}^{E X}}{Z_{1}^{G}}\right) \\
= & \frac{1}{1-n}\left(\log \left\langle\mathcal{O}^{\dagger}\left(r_{l}, \theta_{l}^{n}\right) \mathcal{O}\left(r_{e}, \theta_{e}^{n}\right) \cdots \mathcal{O}^{\dagger}\left(r_{l}, \theta_{l}^{1}\right) \mathcal{O}\left(r_{e}, \theta_{e}^{1}\right)\right\rangle_{\Sigma_{n}}\right. \\
& \left.-n \log \left\langle\mathcal{O}\left(r_{e}, \theta_{e}^{1}\right) \mathcal{O}^{\dagger}\left(r_{l}, \theta_{l}^{1}\right)\right\rangle_{\Sigma_{1}}\right) .
\end{aligned}
$$

In this way, we can express the difference of (Rényi) entanglement entropy using the correlation function on $n$-sheeted manifold.

\section{Propagator}

In this section, we derive the propagator on $n$-sheeted manifold $\Sigma_{n}$. The time evolution of the Rényi entropy after the excitation by the insertion of local operators is given by the 
correlation functions of local operators in the replica space. As in the bosonic case [23], we first compute the propagators on the $n$-sheeted replica space $\Sigma_{n}$ in the Euclidean signature and then analytically continue them in the Lorentzian signature. Propagator of spinor fields on the space with conical singularity is studied in [38]. Because finally we take the $\epsilon \rightarrow 0$ limit, we only consider the propagator which survive in this limit, which we call dominant propagator. Finally, one technical subtlety in the fermionic case is that the fermion fields and the gamma matrices are sensitive to which frame one is using. The frame used in [38], which is suitable in the derivation of propagator in the polar coordinates, is different from the one that are physically interesting and defined in the canonical frame in the Cartesian coordinates, we change the frame to the frame which is canonical in the Cartesian coordinate.

\subsection{Computation of propagator}

In conformal field theories, (Rényi) entanglement entropy for a ground state is invariant under the conformal transformation. In order to preserve its conformal symmetry in the replica trick, the action on $\Sigma_{n}$ is given by

$$
S=\int_{\Sigma_{n}} d V \bar{\psi} \Gamma^{\mu} \nabla_{\mu} \psi
$$

where $\Gamma^{i}(i=0,1,2,3)$ obeys the clifford algebra $\left(\left\{\Gamma^{i}, \Gamma^{j}\right\}=2 \delta_{i, j} \mathbf{1}\right)$ and $\mathbf{1}$ is the identity.

In this case, $\Sigma_{n}$ is given by the flat space except for the origin. We introduce a polar coordinate and this geometry is described by

$$
d s^{2}=d r^{2}+r^{2} d \theta^{2}+d x_{2}^{2}+d x_{3}^{2}
$$

To define the spinor on curved spacetime, we choose a frame. We choose the frame that has the component

$$
e_{0}^{\mu}=\left(\frac{1}{r}, 0, \cdots, 0\right), \quad e_{a}^{\mu}=\delta_{a}^{\mu} \text { for }(a \neq 1)
$$

This is the same choice written in [38]. With this choice, each base vector of local frame $e^{a}$ is directed to the same direction with each base vector with $\partial_{\mu}$ in polar coordinate. The two point function of $\psi$ and $\bar{\psi}$ is defined by

$$
-\left\langle\mathcal{T} \psi_{a}(r, \theta, \mathbf{x}) \bar{\psi}_{b}\left(r_{2}, \theta_{2}, \mathbf{x}_{2}\right)\right\rangle=S_{a b}\left(r, r_{2}, \theta, \theta_{2}, \mathbf{x}, \mathbf{x}_{2}\right) .
$$

$S_{a b}$ obeys the equation of motion as follows,

$$
\left(e_{i}^{\mu} \Gamma^{i} \partial_{\mu}+\frac{\Gamma^{0}}{2 r}\right) S\left(r, r_{2}, \theta, \theta_{2}, \mathbf{x}, \mathbf{x}_{2}\right)=-\mathbf{1} \frac{\delta\left(r-r_{2}\right) \delta\left(\theta-\theta_{2}\right) \delta\left(\mathbf{x}-\mathbf{x}_{\mathbf{2}}\right)}{r} .
$$

$S_{a b}$ can be rewritten by

$$
S_{a b}=\left(e_{i}^{\mu} \Gamma^{i} \partial_{\mu}+\frac{\Gamma^{0}}{2 r}\right)_{a b} g\left(r, r_{2}, \theta, \theta_{2}, \mathbf{x}, \mathbf{x}_{2}\right) .
$$

$g$ is defined by

$$
g\left(x, x^{\prime}\right)=\mathbf{1} \operatorname{Re} H\left(r, r_{2}, \theta, \theta_{2}, \mathbf{x}, \mathbf{x}_{2}\right)+\Gamma^{0} \Gamma^{1} \operatorname{Im} H\left(r, r_{2}, \theta, \theta_{2}, \mathbf{x}, \mathbf{x}_{2}\right) .
$$


where $H\left(x, x^{\prime}\right)$ is given by

$$
H\left(r, r_{2}, \theta, \theta_{2}, \mathbf{x}, \mathbf{x}_{2}\right)=\exp \left(i \frac{\theta-\theta^{\prime}}{2}\right) G\left(r, r_{2}, \theta, \theta_{2}, \mathbf{x}, \mathbf{x}_{2}\right) .
$$

$G\left(r, r_{2}\right)$ obeys the following equation of motion,

$$
\begin{aligned}
\left(\frac{\partial^{2}}{\partial r^{2}}+\frac{1}{r} \frac{\partial}{\partial r}+\frac{1}{r^{2}} \frac{\partial^{2}}{\partial \theta^{2}}+\frac{\partial^{2}}{\left(\partial x^{2}\right)^{2}}+\frac{\partial^{2}}{\left(\partial x^{3}\right)^{2}}\right) & G\left(r, r_{2}, \theta, \theta_{2}, \mathbf{x}, \mathbf{x}_{2}\right)= \\
& -\frac{\delta\left(r-r_{2}\right) \delta\left(\theta-\theta_{2}\right) \delta\left(\mathbf{x}-\mathbf{x}_{\mathbf{2}}\right)}{r} .
\end{aligned}
$$

Next we consider the boundary condition for $\psi(x) .{ }^{1}$ It is given by,

$$
\psi(r, \theta+2 n \pi, \mathbf{x})=-\psi(r, \theta, \mathbf{x}) .
$$

Then the boundary condition for two point function of $\psi$ is given by

$$
S_{a b}\left(r, r_{2}, \theta+2 n \pi, \theta_{2}, \mathbf{x}, \mathbf{x}^{\prime}\right)=-S_{a b}\left(r, r_{2}, \theta, \theta_{2}, \mathbf{x}, \mathbf{x}^{\prime}\right) .
$$

The boundary condition for the green function $G$ is given by

$$
G\left(r, r_{2}, \theta+2 n \pi, \theta_{2}, \mathbf{x}, \mathbf{x}^{\prime}\right)=e^{i(-n+1) \pi} G\left(r, r_{2}, \theta, \theta_{2}, \mathbf{x}, \mathbf{x}^{\prime}\right) .
$$

Let's compute the propagator $G\left(r, r^{\prime}, \theta, \theta^{\prime}\right)$. It can be expanded by the eigenfunctions $V(r, \theta, \mathbf{x})$ which are defined by

$$
\mathcal{L} V(r, \theta, \mathbf{x})=-\left(k^{2}+\mathbf{k}^{2}\right) V(r, \theta, \mathbf{x}),
$$

where $\mathcal{L}=\left(\frac{\partial^{2}}{\partial r^{2}}+\frac{1}{r} \frac{\partial}{\partial r}+\frac{1}{r^{2}} \frac{\partial^{2}}{\partial \theta^{2}}+\frac{\partial^{2}}{\left(\partial x^{2}\right)^{2}}+\frac{\partial^{2}}{\left(\partial x^{3}\right)^{2}}\right)$.

After expanding it, it is given by

$$
\begin{aligned}
G\left(x, x^{\prime}\right)= & \frac{1}{2 n \pi} \frac{1}{(2 \pi)^{2}} \sum_{l=-\infty}^{\infty} \int d \mathbf{k} \int_{0}^{\infty} d k k \frac{J_{\left|\frac{-n+1+2 l}{2 n}\right|}(k r) J_{\left|\frac{-n+1+2 l}{2 n}\right|}\left(k r^{\prime}\right)}{k^{2}+\mathbf{k}^{2}} \\
& \times e^{i\left(\frac{-n+1+2 l}{2 n}\right)\left(\theta-\theta^{\prime}\right)} e^{i \mathbf{k}\left(\mathbf{x}-\mathbf{x}^{\prime}\right)},
\end{aligned}
$$

where $J_{\nu}(x)$ is the Bessel function of the first kind. We can rewrite this green function as in $[23-25,36-38]$. It is given by

$$
G\left(x, x^{\prime}\right)=\frac{1}{8 \pi^{2} n r r^{\prime}} \sum_{l=-\infty}^{\infty} \frac{e^{-\left|\frac{-n+1+2 l}{2 n}\right| t_{0}+i\left(\frac{-n+1+2 l}{2 n}\right)\left(\theta-\theta^{\prime}\right)}}{\sinh t_{0}},
$$

\footnotetext{
${ }^{1}$ As written in [40], we get the sign from each sheet. Also we need to include the extra minus sign which origin is the same with that of thermal partition function. Finally we get $(-1)^{n}$. But this depends on the choice of frames. In this choice, each sign from each sheet is cancelled by the sign from the rotation of frame and we get this boundary condition because only the minus sign from the trace of fermion remains .By using the map in [39], entanglement entropy for the spherical subsystem is map to the thermal entropy in the hyperbolic space. This boundary condition in (4.10) agrees with the one which is imposed on fermionic field along $S^{1}$ direction.
} 
where $t_{0}$ is defined by

$$
\cosh t_{0}=\frac{r^{2}+r^{\prime 2}+\left|\mathbf{x}-\mathbf{x}^{\prime}\right|^{2}}{2 r r^{\prime}} .
$$

If $n$ is odd, the green function is given by

$$
G\left(x, x^{\prime}\right)=\frac{1}{8 \pi^{2} n r r^{\prime}} \frac{\sinh \frac{t_{0}}{n}}{\sinh t_{0}\left(\cosh \left(\frac{t_{0}}{n}\right)-\cos \left(\frac{\theta-\theta^{\prime}}{n}\right)\right)}
$$

In this case, the boundary condition (4.12) is given by

$$
G(r, \theta+2 n \pi, \mathbf{x})=G(r, \theta, \mathbf{x}) .
$$

The green function given by (4.17) obeys this boundary condition.

If $n$ is even, the green function is

$$
G\left(x, x^{\prime}\right)=\frac{1}{4 \pi^{2} n r r^{\prime}} \frac{\cos \left(\frac{\theta-\theta^{\prime}}{2 n}\right) \sinh \frac{t_{0}}{2 n}}{\sinh t_{0}\left(\cosh \left(\frac{t_{0}}{n}\right)-\cos \left(\frac{\theta-\theta^{\prime}}{n}\right)\right)} .
$$

In this case, boundary condition given by (4.12) is given by

$$
G(r, \theta+2 n \pi, \mathbf{x})=-G(r, \theta, \mathbf{x}) .
$$

\subsection{Analytic continuation to real time}

Up to here, we consider propagators in the Euclidean space. Two local operators are located as in figure 3. We would like to study the time evolution of (Rényi) entanglement entropy. Therefore we perform an analytic continuation to real time as follows,

$$
\begin{aligned}
& \tau_{l}=\epsilon-i t, \\
& \tau_{e}=-\epsilon-i t,
\end{aligned}
$$

where in lorentzian spacetime $\epsilon$ is a cutoff parameter which regulates the divergence when a local operator contacts with another.

After performing it, parameters in Euclidean space are related to those in lorentzian spacetime as follows,

$$
\begin{aligned}
r^{2} & =l^{2}-t^{2}+\epsilon^{2}-2 i \epsilon t, \\
r_{2}^{2} & =l^{2}-t^{2}+\epsilon^{2}+2 i \epsilon t, \\
\cos \left(\theta-\theta_{2}\right) & =\frac{l^{2}-\epsilon^{2}-t^{2}}{V^{2}}, \\
\sin \left(\theta-\theta_{2}\right) & =-\frac{2 \epsilon t}{V^{2}}, \\
V^{2} & =\sqrt{\left(l^{2}-t^{2}+\epsilon^{2}\right)^{2}+4 \epsilon^{2} t^{2}} .
\end{aligned}
$$




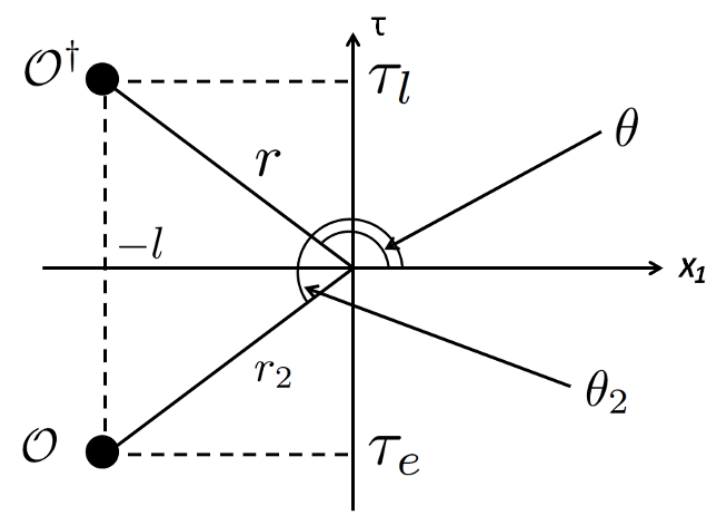

Figure 3. The location of operators in Euclidean space.

\subsection{Dominant propagators}

After performing the analytic continuation in (4.21), we take the limit $\epsilon \rightarrow 0$. A few propagators dominantly contribute to $n$ point functions. We call them dominant propagators. They are $\mathcal{O}\left(\epsilon^{-3}\right)$. There are another propagators which has $\mathcal{O}\left(\epsilon^{-2}\right)$ or less contribution, but they don't contribute when we take $\epsilon \rightarrow 0$ limit. For example, the wick contraction of operators which lives in two or more separated sheets. If $t \leq l$, dominant propagators on $\Sigma_{1}$ are given by

$$
\begin{aligned}
& S_{a b}\left(r, r_{2}, \theta-\theta_{2}\right)=\frac{1}{16 \pi^{2} \sqrt{l^{2}-t^{2}} \epsilon^{3}}\left[i t \Gamma_{a b}^{0}+l \Gamma_{a b}^{1}\right], \\
& S_{a b}\left(r, r_{2}, \theta_{2}-\theta\right)=\frac{-1}{16 \pi^{2} \sqrt{l^{2}-t^{2}} \epsilon^{3}}\left[i t \Gamma_{a b}^{0}+l \Gamma_{a b}^{1}\right] .
\end{aligned}
$$

On the other hand, If $t>l$, they are given by

$$
\begin{aligned}
& S_{a b}\left(r, r_{2}, \theta-\theta_{2}\right)=\frac{1}{16 \pi^{2} \sqrt{t^{2}-l^{2}} \epsilon^{3}}\left[i l \Gamma_{a b}^{0}+t \Gamma_{a b}^{1}\right], \\
& S_{a b}\left(r, r_{2}, \theta_{2}-\theta\right)=\frac{-1}{16 \pi^{2} \sqrt{t^{2}-l^{2}} \epsilon^{3}}\left[i l \Gamma_{a b}^{0}+t \Gamma_{a b}^{1}\right],
\end{aligned}
$$

If $t \leq l$, dominant propagators on $\Sigma_{n>1}$ are red arrows in figure 4 . They are given by

$$
\begin{aligned}
& S_{a b}\left(r, r_{2}, \theta-\theta_{2}\right)=\frac{1}{16 \pi^{2} \sqrt{l^{2}-t^{2}} \epsilon^{3}}\left[i t \Gamma_{a b}^{0}+l \Gamma_{a b}^{1}\right], \\
& S_{a b}\left(r_{2}, r, \theta_{2}-\theta\right)=\frac{-1}{16 \pi^{2} \sqrt{l^{2}-t^{2}} \epsilon^{3}}\left[i t \Gamma_{a b}^{0}+l \Gamma_{a b}^{1}\right] .
\end{aligned}
$$

If $t>l$, they are red arrows and blue arrows in figure 4 . They are given by

$$
\begin{aligned}
S_{a b}\left(r, r_{2}, \theta-\theta_{2}\right) & =\frac{(t+l)^{2}}{64 \pi^{2} t \sqrt{t^{2}-l^{2}} \epsilon^{3}}\left[i \Gamma_{a b}^{0}+\frac{2 t-l}{t} \Gamma_{a b}^{1}\right], \\
S_{a b}\left(r_{2}, r, \theta_{2}-\theta\right) & =\frac{-(t+l)^{2}}{64 \pi^{2} t \sqrt{t^{2}-l^{2}} \epsilon^{3}}\left[i \Gamma_{a b}^{0}+\frac{2 t-l}{t} \Gamma_{a b}^{1}\right], \\
S_{a b}\left(r, r_{2}, \theta-\theta_{2}+2 \pi\right) & =\frac{(t-l)^{2}}{64 \pi^{2} t \sqrt{t^{2}-l^{2}} \epsilon^{3}}\left[i \Gamma_{a b}^{0}-\frac{2 t+l}{t} \Gamma_{a b}^{1}\right],
\end{aligned}
$$



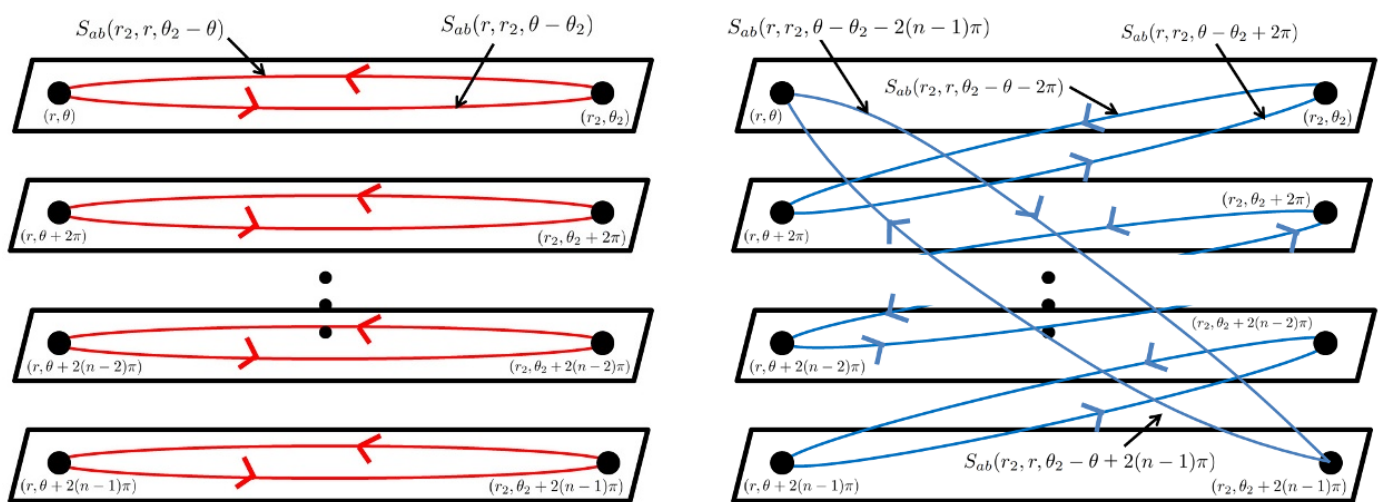

Figure 4. The schematic description of dominant propagators. $S_{a b}\left(r, r_{2}, \theta-\theta_{2}\right)$ and $S_{a b}\left(r_{2}, r, \theta_{2}-\theta\right)$ correspond to the right arrow and the left arrow respectively.

$$
\begin{aligned}
S_{a b}\left(r_{2}, r, \theta_{2}-\theta-2 \pi\right) & =\frac{-(t-l)^{2}}{64 \pi^{2} t \sqrt{t^{2}-l^{2}} \epsilon^{3}}\left[i \Gamma_{a b}^{0}-\frac{2 t+l}{t} \Gamma_{a b}^{1}\right], \\
S_{a b}\left(r, r_{2}, \theta-\theta_{2}-2(n-1) \pi\right) & =\frac{-(t-l)^{2}}{64 \pi^{2} t \sqrt{t^{2}-l^{2}} \epsilon^{3}}\left[i \Gamma_{a b}^{0}-\frac{2 t+l}{t} \Gamma_{a b}^{1}\right], \\
S_{a b}\left(r_{2}, r, \theta_{2}-\theta+2(n-1) \pi\right) & =\frac{(t-l)^{2}}{64 \pi^{2} t \sqrt{t^{2}-l^{2}} \epsilon^{3}}\left[i \Gamma_{a b}^{0}-\frac{2 t+l}{t} \Gamma_{a b}^{1}\right] .
\end{aligned}
$$

\subsubsection{Propagators in canonical frame in the Cartesian coordinate}

Given locally excited states are defined by being acted by local operators on the ground state in canonical frame. ${ }^{2}$ Therefore we explain the relation between propagators in (4.1) and them in the canonical frame. In the Cartesian coordinate, the action in free massless fermionic theory is given by

$$
S=\int d^{4} x \bar{\psi}^{\prime} \gamma^{\mu} \partial_{\mu} \psi^{\prime}
$$

where $\gamma^{\mu}=\left\{\gamma^{0}, \gamma^{1}, \gamma^{2}, \gamma^{3}\right\}$.

When we introduce a polar coordinate $x^{1}=r \cos \theta, x^{0}=r \sin \theta$ and redefine the fermionic field by $\psi^{\prime}(r, \theta)=e^{-\frac{\gamma^{1} \gamma^{0}}{2} \theta} \psi(r, \theta)$, it is rewritten by

$$
S=\int d r \int d \theta r \int d^{2} \mathbf{x} \bar{\psi}\left[\Gamma^{0} \partial_{r}+\Gamma^{1} \frac{\partial_{\theta}}{r}+\frac{\Gamma^{0}}{2 r}+\Gamma^{\mathbf{x}} \partial_{\mathbf{x}}\right] \psi
$$

where $\Gamma^{0}=\gamma^{1}, \Gamma^{1}=\gamma^{0}, \Gamma^{\mathbf{x}}=\gamma^{\mathbf{x}}$.

$$
\psi^{\prime}(r, \theta)=\cos \left(\frac{\theta}{2}\right) \psi(r, \theta)+\sin \left(\frac{\theta}{2}\right) \gamma^{0} \gamma^{1} \psi(r, \theta) .
$$

After performing this map, the boundary condition for $\psi^{\prime 3}$ is mapped to

$$
\psi^{\prime}(r, \theta+2 n \pi)=(-1)^{n+1} \psi^{\prime}(r, \theta) .
$$

\footnotetext{
2 "Canonical frame" means the frame given by $e_{\mu}^{a}=\delta_{\mu}^{a}$ in Cartesian coordinate in flat spacetime.

${ }^{3}$ When $n=1, \psi^{\prime}$ is regular at the origin.
} 
We define propagators by

$$
\begin{aligned}
-\left\langle\mathcal{T} \psi_{a}^{\prime}(r, \theta) \bar{\psi}_{b}^{\prime}\left(r_{2}, \theta_{2}\right)\right\rangle & =S_{a b}^{\prime}\left(r, r_{2}, \theta-\theta_{2}\right) \\
-\left\langle\mathcal{T} \psi_{a}^{\prime}(r, \theta) \psi_{b}^{\dagger \dagger}\left(r_{2}, \theta_{2}\right)\right\rangle & =S_{a c}^{\prime}\left(r, r_{2}, \theta-\theta_{2}\right) \gamma_{c b}^{0}=V_{a b}\left(r, r_{2}, \theta-\theta_{2}\right) .
\end{aligned}
$$

After performing this map, we also perform the analytic continuation in (4.21) and take the limit $\epsilon \rightarrow 0$. After that, only a few propagators can contribute to $n$ point function dominantly. They are $\mathcal{O}\left(\epsilon^{-3}\right)$. In any time, dominant propagators on $\Sigma_{1}$ are given by

$$
\begin{aligned}
S_{a b}^{\prime}\left(r, r_{2}, \theta-\theta_{2}\right) & =\frac{-i}{16 \pi^{2} \epsilon^{3}} \gamma_{a b}^{t}, \\
S_{a b}^{\prime}\left(r_{2}, r, \theta_{2}-\theta\right) & =\frac{i}{16 \pi^{2} \epsilon^{3}} \gamma_{a b}^{t}, \\
V_{a b}\left(r, r_{2}, \theta-\theta_{2}\right) & =\frac{-1}{16 \pi^{2} \epsilon^{3}} \mathbf{1}_{a b}, \\
V_{a b}\left(r_{2}, r, \theta_{2}-\theta\right) & =\frac{1}{16 \pi^{2} \epsilon^{3}} \mathbf{1}_{a b},
\end{aligned}
$$

where $\mathbf{1}$ is identity. $\gamma^{0}$ is changed to $i \gamma^{t}\left(\left(\gamma^{t}\right)^{2}=-\mathbf{1}\right)$.

On the other hand, if $t \leq l$ those on $\Sigma_{n>1}$ are the same as in (4.32). In the region $t>l$, they are given by

$$
\begin{aligned}
S_{a b}^{\prime}\left(r, r_{2}, \theta-\theta_{2}\right) & =\frac{-i(t+l)}{64 \pi^{2} t \epsilon^{3}}\left[\frac{t-l}{t} \gamma_{a b}^{1}+2 \gamma_{a b}^{t}\right], \\
S_{a b}^{\prime}\left(r_{2}, r, \theta_{2}-\theta\right) & =\frac{i(t+l)}{64 \pi^{2} t \epsilon^{3}}\left[\frac{t-l}{t} \gamma_{a b}^{1}+2 \gamma_{a b}^{t}\right], \\
S_{a b}^{\prime}\left(r, r_{2}, \theta-\theta_{2}+2 \pi\right) & =\frac{i(t-l)}{64 \pi^{2} t \epsilon^{3}}\left[\frac{t+l}{t} \gamma_{a b}^{1}-2 \gamma_{a b}^{t}\right], \\
S_{a b}^{\prime}\left(r_{2}, r, \theta_{2}-\theta-2 \pi\right) & =-\frac{i(t-l)}{64 \pi^{2} t \epsilon^{3}}\left[\frac{t+l}{t} \gamma_{a b}^{1}-2 \gamma_{a b}^{t}\right], \\
S_{a b}^{\prime}\left(r, r_{2}, \theta-\theta_{2}-2(n-1) \pi\right) & =(-1)^{n-1} \frac{i(t-l)}{64 \pi^{2} t \epsilon^{3}}\left[\frac{t+l}{t} \gamma_{a b}^{1}-2 \gamma_{a b}^{t}\right], \\
S_{a b}^{\prime}\left(r_{2}, r, \theta_{2}-\theta+2(n-1) \pi\right) & =(-1)^{n} \frac{i(t-l)}{64 \pi^{2} t \epsilon^{3}}\left[\frac{t+l}{t} \gamma_{a b}^{1}-2 \gamma_{a b}^{t}\right],
\end{aligned}
$$

and

$$
\begin{aligned}
V_{a b}\left(r, r_{2}, \theta-\theta_{2}\right) & =-\frac{(t+l)}{64 \pi^{2} t \epsilon^{3}}\left[-\frac{t-l}{t}\left(\gamma^{1} \gamma^{t}\right)_{a b}+2 \mathbf{1}_{a b}\right], \\
V_{a b}\left(r_{2}, r, \theta_{2}-\theta\right) & =\frac{(t+l)}{64 \pi^{2} t \epsilon^{3}}\left[-\frac{t-l}{t}\left(\gamma^{1} \gamma^{t}\right)_{a b}+2 \mathbf{1}_{a b}\right], \\
V_{a b}\left(r, r_{2}, \theta-\theta_{2}+2 \pi\right) & =\frac{(t-l)}{64 \pi^{2} t \epsilon^{3}}\left[-\frac{t+l}{t}\left(\gamma^{1} \gamma^{t}\right)_{a b}-2 \mathbf{1}_{a b}\right], \\
V_{a b}\left(r_{2}, r, \theta_{2}-\theta-2 \pi\right) & =-\frac{(t-l)}{64 \pi^{2} t \epsilon^{3}}\left[-\frac{t+l}{t}\left(\gamma^{1} \gamma^{t}\right)_{a b}-2 \mathbf{1}_{a b}\right], \\
V_{a b}\left(r, r_{2}, \theta-\theta_{2}-2(n-1) \pi\right) & =(-1)^{n-1} \frac{(t-l)}{64 \pi^{2} t \epsilon^{3}}\left[-\frac{t+l}{t}\left(\gamma^{1} \gamma^{t}\right)_{a b}-2 \mathbf{1}_{a b}\right], \\
V_{a b}\left(r_{2}, r, \theta_{2}-\theta+2(n-1) \pi\right) & =(-1)^{n} \frac{(t-l)}{64 \pi^{2} t \epsilon^{3}}\left[-\frac{t+l}{t}\left(\gamma^{1} \gamma^{t}\right)_{a b}-2 \mathbf{1}_{a b}\right] .
\end{aligned}
$$

Let's study the time evolution of $\Delta S_{A}^{(n)}$ in the next section. 


\section{$5 \Delta S_{A}^{(n)}$ for various local operators}

In this section, we study the time evolution of $\Delta S_{A}^{(n)}$ for various operators by the replica trick. Especially, we focus on their behavior in the late time region $(t \gg l)$.

\section{$5.1 \Delta S_{A}^{(n)}$ for $\psi_{a}^{\prime}$}

The locally exited state is given by

$$
|\Psi\rangle=\mathcal{N} \psi_{a}^{\prime}(-t,-l, \mathbf{x})|0\rangle .
$$

The time evolution of $\Delta S_{A}^{(n)}$ is as follows.

\subsubsection{The excess of Rényi entanglement entropies}

$\Delta S_{A}^{(n)}$ for it is given by

$$
\Delta S_{A}^{(n)}=\frac{1}{1-n} \log \left[\frac{\left\langle\psi_{a}^{\prime \dagger}(\theta+2(n-1) \pi) \psi_{a}^{\prime}\left(\theta_{2}+2(n-1) \pi\right) \cdots \psi_{a}^{\prime \dagger}(\theta) \psi_{a}^{\prime}\left(\theta_{2}\right)\right\rangle_{\Sigma_{n}}}{\left\langle\psi_{a}^{\prime \dagger}(\theta) \psi_{a}^{\prime}\left(\theta_{2}\right)\right\rangle_{\Sigma_{1}}^{n}}\right] .
$$

When we take the limit $\epsilon \rightarrow 0, \Delta S_{A}^{(n)}$ vanishes in the early time region $(t \leq l)$. When $t$ is greater than $l$, two diagram in figure 5 dominantly contribute to $\Delta S_{A}^{(n)}$. In this region the denominator is given by

$$
\left\langle\psi_{a}(r, \theta)^{\dagger} \psi_{a}\left(r_{2}, \theta_{2}\right)\right\rangle_{\Sigma_{1}}^{n} \sim\left(\frac{1}{16 \pi^{2} \epsilon^{3}}\right)^{n}
$$

Therefore $\Delta S_{A}^{(n)}$ is given by $\mathrm{f}$

$$
\begin{aligned}
\Delta S_{A}^{(n)} & =\frac{1}{1-n} \log \left[A_{1}+A_{2}\right], \\
A_{1} & =\left(\frac{t+l}{4 t}\right)^{n}\left(\left(\frac{t-l}{t}\right)\left(\gamma^{t} \gamma^{1}\right)_{a a}+2\right)^{n}, \\
A_{2} & =\left(\frac{t-l}{4 t}\right)^{n}\left(-\left(\frac{t+l}{t}\right)\left(\gamma^{t} \gamma^{1}\right)_{a a}+2\right)^{n},
\end{aligned}
$$

where $\left(\gamma^{t} \gamma^{1}\right)_{a a}$ is real because $\gamma^{t} \gamma^{1}$ is a hermitian matrix. ${ }^{4}$ If we take the late time limit $(t \gg l), \Delta S_{A}^{(n)}$ is given by

$$
\begin{aligned}
\Delta S_{A}^{(n)} & =\frac{1}{1-n} \log \left[A_{1}+A_{2}\right], \\
A_{1} & =\left(\frac{\left(\gamma^{t} \gamma^{1}\right)_{a a}+2}{4}\right)^{n}, \\
A_{2} & =\left(\frac{-\left(\gamma^{t} \gamma^{1}\right)_{a a}+2}{4}\right)^{n},
\end{aligned}
$$

\footnotetext{
${ }^{4}-1 \leq\left(\gamma^{t} \gamma^{1}\right)_{a a} \leq 1$.
} 


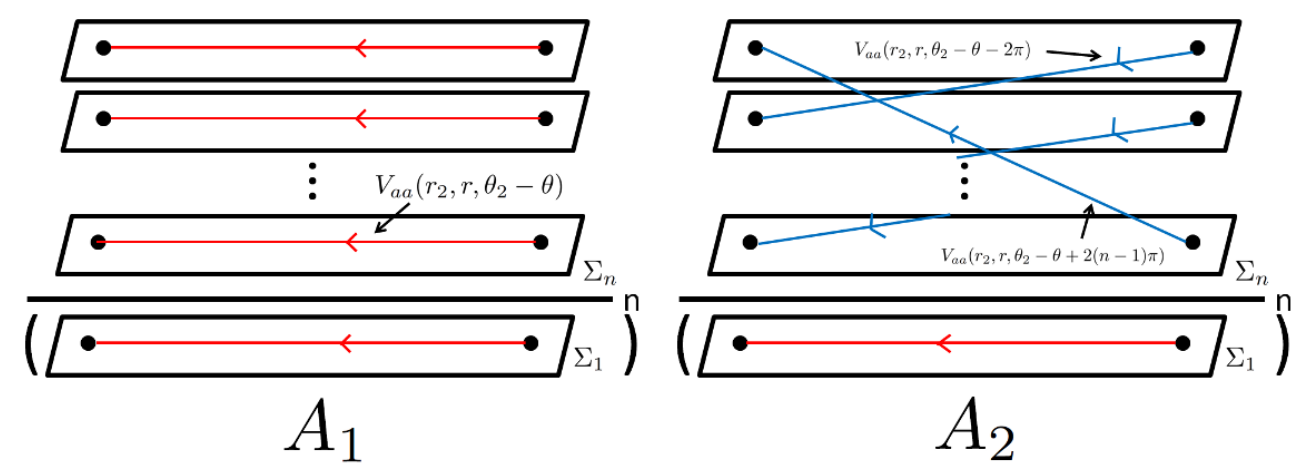

Figure 5. The schematic description of $A_{1}$ and $A_{2}$.

where as in figure $5, A_{1}$ and $A_{2}$ are respectively given by

$$
\begin{aligned}
A_{1} & =\frac{\left(V_{a a}\left(r_{2}, r, \theta_{2}-\theta\right)\right)^{n}}{\left\langle\psi_{a}^{\prime \dagger}(r, \theta) \psi_{a}^{\prime}\left(r_{2}, \theta_{2}\right)\right\rangle_{\Sigma_{1}}^{n}}, \\
A_{2} & =\frac{(-1)^{n+1}\left(V_{a a}\left(r_{2}, r, \theta_{2}-\theta-2 \pi\right)\right)^{n-1} V_{a a}\left(r_{2}, r, \theta_{2}-\theta+2(n-1) \pi\right)}{\left\langle\psi_{a}^{\prime \dagger}(r, \theta) \psi_{a}^{\prime}\left(r_{2}, \theta_{2}\right)\right\rangle_{\Sigma_{1}}^{n}} .
\end{aligned}
$$

If we take the Von Neumann limit $n \rightarrow 1, \Delta S_{A}$ is given by

$$
\Delta S_{A}=\frac{1}{4}\left(\left(\gamma^{t} \gamma^{1}\right)_{a a}-2\right) \log \left(2-\left(\gamma^{t} \gamma^{1}\right)_{a a}\right)-\frac{1}{4}\left(\left(\gamma^{t} \gamma^{1}\right)_{a a}+2\right) \log \left(\left(\gamma^{t} \gamma^{1}\right)_{a a}+2\right)+\log (4) .
$$

If $\left(\gamma^{t} \gamma^{1}\right)_{a a}$ vanishes, $\Delta S_{A}$ is given by $\log 2$ which is entanglement entropy for the EPR state. The lower value of $\Delta S_{A}$ in (5.7) is given by $\log 4 / 3^{\frac{3}{4}}\left(\left(\gamma^{t} \gamma^{1}\right)_{a a}=1\right.$ or -1$)$.

\subsubsection{Reduced density matrix}

In this section, we assume that the execss of (Rényi) entanglement entropy can be understood as the (Rényi) entanglement entropy of finite dimensional quantum system made from quasi-particle excitation, as in [23]. In other words, we assume that we can separate the (Rényi) entanglement entropy as the summation of ground state contribution and quasi-particle contribution. If we identify $A_{1}$ and $A_{2}$ as the diagonal components of $\rho_{A}^{n}$ respectively, it is expected that the density matrix for this state is given by

$$
\rho_{A}=\frac{1}{4}\left(\begin{array}{cc}
\left(\gamma^{t} \gamma^{1}\right)_{a a}+2 & 0 \\
0 & -\left(\gamma^{t} \gamma^{1}\right)_{a a}+2
\end{array}\right),
$$

where $\operatorname{tr}_{A} \rho_{A}$ and each diagonal components of $\rho_{A}$ are positive.

It is expected that the density matrix for $|\Psi\rangle=\mathcal{N} \bar{\psi}_{a}^{\prime}|0\rangle$ is given by

$$
\rho_{A}=\frac{1}{4}\left(\begin{array}{cc}
\left(\gamma^{1} \gamma^{t}\right)_{a a}+2 & 0 \\
0 & -\left(\gamma^{1} \gamma^{t}\right)_{a a}+2
\end{array}\right) .
$$




\section{$5.2 \Delta S_{A}^{(n)}$ for $\bar{\psi}^{\prime} \psi^{\prime}$}

A locally excited state is given by

$$
|\Psi\rangle=\mathcal{N} \bar{\psi}^{\prime} \psi^{\prime}(-t,-l, \mathbf{x})|0\rangle,
$$

which is a scalar for $\operatorname{SL}(2, \mathbf{C})$ transformation.

Let's study the time evolution of $\Delta S_{A}^{(n)}$.

\subsubsection{The excess of (Rényi) entanglement entropy}

$\Delta S_{A}^{(2)}$ for (5.10) is given by

$$
\Delta S_{A}^{(2)}=-\log \left[\frac{\left\langle\bar{\psi} \psi(\theta+2 \pi) \bar{\psi} \psi\left(\theta_{2}+2 \pi\right) \bar{\psi} \psi(\theta) \bar{\psi} \psi\left(\theta_{2}\right)\right\rangle_{\Sigma_{2}}}{\left\langle\bar{\psi} \psi(\theta) \bar{\psi} \psi\left(\theta_{2}\right)\right\rangle_{\Sigma_{1}}^{2}}\right]
$$

In the early time region $(t \leq l), \Delta S_{A}^{(2)}$ vanishes when we take the limit $\epsilon \rightarrow 0$. On the other hand, in the region $t>l, \Delta S_{A}^{(2)}$ is given by

$$
\begin{aligned}
\Delta S_{A}^{(2)} & =-\log \left[A_{1}+A_{2}+A_{3}\right], \\
A_{1} & =\left[\frac{c(t+l)^{4}}{2^{4} c t^{2}\left(t^{2}-l^{2}\right)}\left[\left(\frac{2 t-l}{t}\right)^{2}-1\right]\right]^{2}, \\
A_{2} & =\left[\frac{c(t-l)^{4}}{2^{4} c t^{2}\left(t^{2}-l^{2}\right)}\left[\left(\frac{2 t+l}{t}\right)^{2}-1\right]\right]^{2}, \\
A_{3} & =2 c\left[\left(\frac{t^{2}-l^{2}}{2^{4} t^{2} c}\right)^{2}\left[\left(\frac{5 t^{2}-l^{2}}{t^{2}}\right)^{2}+16\right]\right],
\end{aligned}
$$

where $c=\operatorname{Tr} 1$ is a trace of identity for Dirac spinor induces and in this case this is given by $c=4$.

If we take the limit $t \rightarrow \infty, \Delta S_{A}^{(2)}$ is given by

$$
\Delta S_{A}^{(2)}=\log \left[\frac{2^{9}}{77}\right]
$$

If $t \leq l$, for arbitrary $n, \Delta S_{A}^{(n)}$ vanishes. In the region $t>l, \Delta S_{A}^{(n)}$ is given by

$$
\Delta S_{A}^{(n)}=\frac{1}{1-n} \log \left[A_{1}+A_{2}+A_{3}\right]
$$

where $A_{1}, A_{2}, A_{3}$ are given by

$$
\begin{aligned}
& A_{1}=\left[\frac{c(t+l)^{4}}{2^{4} c t^{2}\left(t^{2}-l^{2}\right)}\left[\left(\frac{2 t-l}{t}\right)^{2}-1\right]\right]^{n} \\
& A_{2}=\left[\frac{c(t-l)^{4}}{2^{4} c t^{2}\left(t^{2}-l^{2}\right)}\left[\left(\frac{2 t+l}{t}\right)^{2}-1\right]\right]^{n} \\
& A_{3}=2 c\left(\frac{t^{2}-l^{2}}{2^{4} t^{2} c}\right)^{n}\left[\sum_{k \in 2 \mathbb{Z}}^{k \leq n}\left(\begin{array}{l}
n \\
k
\end{array}\right) 4^{k}\left(\frac{5 t^{2}-l^{2}}{t^{2}}\right)^{n-k}\right] .
\end{aligned}
$$


If we take the late time limit $t \rightarrow \infty, \Delta S_{A}^{(n)}$ is given by

$$
\Delta S_{A}^{(n)}=\frac{1}{1-n} \log \left[2\left(\frac{12}{64}\right)^{n}+8\left(\frac{1}{64}\right)^{n} \sum_{k \in 2 \mathbb{Z}}^{k \leq n}\left(\begin{array}{l}
n \\
k
\end{array}\right) 4^{k} 5^{n-k}\right] .
$$

\subsubsection{Reduced density matrix}

By using results up to here, we are able to guess the reduce density matrix for $|\Psi\rangle=\mathcal{N} \bar{\psi}^{\prime} \psi^{\prime}$ as follows. In the limit $\epsilon \rightarrow 0$, only four diagrams in figure 6 can contribute to $\Delta S_{A}^{(n)}$. We define $A_{1}, A_{2}, A$ by

$$
\begin{aligned}
& A_{1}=\frac{12}{64}, \\
& A_{2}=\frac{12}{64}, \\
& A=\frac{1}{64}\left(\begin{array}{ll}
5 & 4 \\
4 & 5
\end{array}\right) .
\end{aligned}
$$

In the replica trick, $\left(A_{1}\right)^{n},\left(A_{2}\right)^{n}$ and $8 \cdot \operatorname{tr}\left(A^{n}\right)$ respectively correspond to the red-lined diagram, blue lined diagram and green lined diagrams in figure.6. Therefore it is expected that the reduced density matrix for this state is given by the $10 \times 10$ matrix,

$$
\rho_{A}=\frac{1}{64}\left(\begin{array}{cccccc}
12 & 0 & 0 & 0 & 0 & 0 \\
0 & \tilde{A} & 0 & 0 & 0 & 0 \\
0 & 0 & \tilde{A} & 0 & 0 & 0 \\
0 & 0 & 0 & \tilde{A} & 0 & 0 \\
0 & 0 & 0 & 0 & \tilde{A} & 0 \\
0 & 0 & 0 & 0 & 0 & 12
\end{array}\right),
$$

where $\tilde{A}$ is given by the $2 \times 2$ matrix,

$$
\tilde{A}=\left(\begin{array}{ll}
5 & 4 \\
4 & 5
\end{array}\right)
$$

If we diagonalize the matrix $\tilde{A}$, it is given by

$$
\tilde{A}=\left(\begin{array}{ll}
9 & 0 \\
0 & 1
\end{array}\right) \text {. }
$$

For any $n$, (Rényi) entanglement entropy is given by

$$
\Delta S_{A}^{(n)}=\frac{1}{1-n} \log \left[\frac{2 \cdot 12^{n}+4 \cdot 9^{n}+4}{2^{6 n}}\right]
$$

If we take the von Neumann entropy limit $n \rightarrow 1$, entanglement entropy is given by

$$
\Delta S_{A}=\frac{3}{4} \log \left(\frac{128}{9}\right)
$$



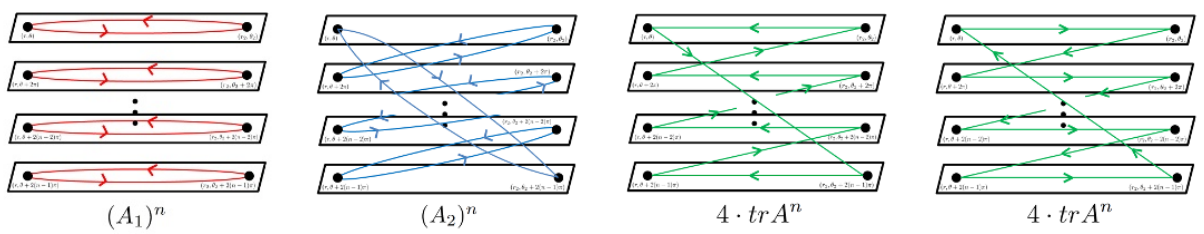

Figure 6. The schematic explanation of the correspondence between diagrams and components of a reduce density matrix.

Min-entropy ${ }^{5}$ is given by

$$
\Delta S_{A}^{(\infty)}=\log \left(\frac{16}{3}\right)
$$

(Rényi) entanglement entropy for this state monotonically decreases when the replica number $n$ increases. Therefore we can consider $\Delta S_{A}^{(\infty)}$ as the lower bound of (Rényi) entanglement entropies.

If we take the large $N$ limit in the $\mathrm{U}(N)$ or $\mathrm{SU}(N)$ free massless fermionic field theory, the number of diagrams which can dominantly contribute to $\Delta S_{A}^{(n)}$ decreases. Because the number of trace in the green-lined diagram is less than that in the others, the blue-lined and red-lined diagram dominantly contribute to $\Delta S_{A}^{(n)}$ in the large $N$ limit. Therefore it is given by

$$
\Delta S_{A}^{(n \geq 2)}=\frac{2 n-1}{n-1} \log 2+\frac{n}{1-n} \log \left(\frac{3}{4}\right) .
$$

After that, if we take $n \rightarrow \infty$ limit, Min-entropy is given by

$$
\Delta S_{A}^{(\infty)}=\log \left(\frac{16}{3}\right)
$$

This result describes that Min-entropy is same as (5.25) even if we take the large $N$ limit.

\section{$5.3 \Delta S_{A}^{(n)}$ for $\psi^{\prime \dagger} \psi^{\prime}$}

A locally excited state is given by

$$
|\Psi\rangle=\mathcal{N} \psi^{\prime \dagger} \psi^{\prime}(-t,-l, \mathbf{x})|0\rangle
$$

which is variant under the $\mathrm{SL}(2, \mathbf{C})$ transformation.

\subsubsection{The excess of (Rényi) entanglement entropy}

$\Delta S_{A}^{(2)}$ for the state in (5.28) is given by

$$
\Delta S_{A}^{(2)}=-\log \left[\frac{\left\langle\psi^{\prime \dagger} \psi^{\prime}(\theta+2 \pi) \psi^{\prime \dagger} \psi^{\prime}\left(\theta_{2}+2 \pi\right) \psi^{\prime \dagger} \psi^{\prime}(\theta) \psi^{\prime \dagger} \psi^{\prime}\left(\theta_{2}\right)\right\rangle_{\Sigma_{2}}}{\left\langle\psi^{\prime \dagger} \psi^{\prime}(\theta) \psi^{\prime \dagger} \psi^{\prime}\left(\theta_{2}\right)\right\rangle_{\Sigma_{1}}^{2}}\right]
$$

\footnotetext{
${ }^{5}$ Here Min-entropy is defined by $\lim _{n \rightarrow \infty} S_{A}^{(n)}$.
} 
If we take the limit $\epsilon \rightarrow 0, \Delta S_{A}^{(2)}$ vanishes in the early time region $(t \leq l)$. In the region $t>l, \Delta S_{A}^{(2)}$ nontrivially grows as follows,

$$
\begin{aligned}
\Delta S_{A}^{(2)} & =-\log \left[A_{1}+A_{2}+A_{3}+A_{4}\right], \\
A_{1} & =\frac{(t+l)^{4}}{4^{6} t^{4}}\left(16+4\left(\frac{t-l}{t}\right)^{2}\right)^{2}, \\
A_{2} & =\frac{(t-l)^{4}}{4^{6} t^{4}}\left(16+4\left(\frac{t+l}{t}\right)^{2}\right)^{2}, \\
A_{3} & =\frac{\left(t^{2}-l^{2}\right)^{2}}{4^{6} t^{4}}\left(4\left(\frac{t^{2}-l^{2}}{t^{2}}-4\right)^{2}+4 \times \frac{4 l^{2}}{t^{2}}\right), \\
A_{4} & =\frac{\left(t^{2}-l^{2}\right)^{2}}{4^{6} t^{4}}\left(4\left(\frac{t^{2}-l^{2}}{t^{2}}-4\right)^{2}+4 \times \frac{4 l^{2}}{t^{2}}\right) .
\end{aligned}
$$

If we take the late time limit $(t \gg l), \Delta S_{A}^{(2)}$ is given by

$$
\Delta S_{A}^{(2)}=-\log \left[\frac{20^{2}}{4^{6}}+\frac{20^{2}}{4^{6}}+\frac{4 \cdot 3^{2}}{4^{6}}+\frac{4 \cdot 3^{2}}{4^{6}}\right] .
$$

If $t \leq l$, for arbitrary $n, \Delta S_{A}^{(n)}$ vanishes. In the region $t>l, \Delta S_{A}^{(n)}$ is given by

$$
\Delta S_{A}^{(n)}=\frac{1}{1-n} \log \left[A_{1}+A_{2}+A_{3}\right]
$$

where $A_{1}, A_{2}, A_{3}$ are given by

$$
\begin{aligned}
& A_{1}=\left[\frac{c(t+l)^{2}}{2^{4} c t^{2}}\left[\left(\frac{t-l}{t}\right)^{2}+4\right]\right]^{n} \\
& A_{2}=\left[\frac{c(t-l)^{2}}{2^{4} c t^{2}}\left[\left(\frac{t+l}{t}\right)^{2}+4\right]\right]^{n} \\
& A_{3}=2 c\left(\frac{t^{2}-l^{2}}{2^{4} t^{2} c}\right)^{n}\left[\sum_{k \in 2 \mathbb{Z}}^{k \leq n}\left(\begin{array}{l}
n \\
k
\end{array}\right)\left(\frac{4 l}{t}\right)^{k}\left(\frac{3 t^{2}+l^{2}}{t^{2}}\right)^{n-k}\right] .
\end{aligned}
$$

If we take the late time limit $t \rightarrow \infty, \Delta S_{A}^{(n)}$ is given by

$$
\Delta S_{A}^{(n)}=\frac{1}{1-n} \log \left[2\left(\frac{20}{64}\right)^{n}+8\left(\frac{3}{64}\right)^{n}\right] .
$$

\subsection{Reduced density matrix}

We define $B_{1}, B_{2}, B$ by

$$
\begin{aligned}
& B_{1}=\frac{20}{64}, \\
& B_{2}=\frac{20}{64}, \\
& B=\frac{1}{64}\left(\begin{array}{ll}
3 & 0 \\
0 & 3
\end{array}\right) .
\end{aligned}
$$


In the replica trick, $\left(B_{1}\right)^{n},\left(B_{2}\right)^{n}$ and $8 \cdot \operatorname{tr}\left(B^{n}\right)$ respectively correspond to the red dashed lined diagram, blue dashed lined diagram and green dashed lined diagrams in figure 7 . Therefore it is expected that the reduced density matrix for this state is given by the $10 \times 10$ matrix,

$$
\rho_{A}=\frac{1}{2^{6}}\left(\begin{array}{cccccc}
20 & 0 & 0 & 0 & 0 & 0 \\
0 & \tilde{B} & 0 & 0 & 0 & 0 \\
0 & 0 & \tilde{B} & 0 & 0 & 0 \\
0 & 0 & 0 & \tilde{B} & 0 & 0 \\
0 & 0 & 0 & 0 & \tilde{B} & 0 \\
0 & 0 & 0 & 0 & 0 & 20
\end{array}\right)
$$

where $\tilde{B}$ is given by $2 \times 2$ matrix,

$$
\tilde{B}=\left(\begin{array}{ll}
3 & 0 \\
0 & 3
\end{array}\right) .
$$

If we take Von Neumann entropy limit $n \rightarrow 1$ in (5.34) entanglement entropy is given by

$$
\Delta S_{A}=\log 16+\frac{3}{4} \log 2-\frac{3}{8} \log 3-\frac{5}{8} \log 5
$$

Although the local operator $\psi^{\prime \dagger} \psi^{\prime}$ is not a scalar under $\operatorname{SL}(2, \mathbf{C})$ transformation, $\Delta S_{A}^{(n)}$ is invariant under this transformation.

If we take the limit $n \rightarrow \infty$, it is given by

$$
\Delta S_{A}^{(\infty)}=\log \left(\frac{16}{5}\right)
$$

If we take the large $N$ limit in the $\mathrm{U}(N)$ or $\mathrm{SU}(N)$ free massless fermionic field theory, the number of diagrams which can contribute to $\Delta S_{A}^{(n)}$ decreases. Because the number of trace in green dashed lined diagram is less than that in the others, blue dashed lined and red dashed lined diagram dominantly contribute to $\Delta S_{A}^{(n)}$ in the large $N$ limit. Therefore it is given by

$$
\Delta S_{A}^{(n)}=\frac{1}{1-n} \log 2+\frac{n}{1-n} \log \left(\frac{5}{16}\right)
$$

If we take $n \rightarrow \infty$ limit, $\Delta S_{A}^{(\infty)}$ is given by

$$
\Delta S_{A}^{(\infty)}=\log \left(\frac{16}{5}\right)
$$

Even if we take the large $N$ limit, the lower bound of $\Delta S_{A}^{(n)}$ agrees with that in (5.39) similarly to the result in the previous subsection.

\subsection{Spin dependence}

Here we discuss the spin dependence of the reduced density matrices in (5.8) and (5.9). Their components have $\left(\gamma^{t} \gamma^{1}\right)_{a a}$. Therefore they depend on the direction of the spin. 

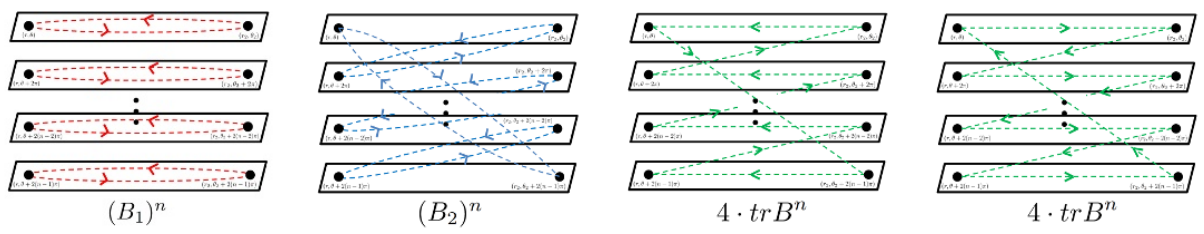

Figure 7. The schematic explanation of the correspondence between diagrams and components of a reduce density matrix.

These spin dependences can be understood as follows. To explain why matrix components depend on the spin, we diagonalize $\gamma^{t} \gamma^{1}$ and it is given by

$$
\gamma^{t} \gamma^{1}=\left(\begin{array}{cccc}
1 & 0 & 0 & 0 \\
0 & -1 & 0 & 0 \\
0 & 0 & 1 & 0 \\
0 & 0 & 0 & -1
\end{array}\right)
$$

We can derive $\left(p_{0}+\gamma^{t} \gamma^{i} p_{i}\right) \Phi(p)=0$ from the equation of motion for $\Phi(p)$ where $\Phi(p)$ are the solution of Dirac equation on the plane with momentum $p$. The modes propagating along $x^{1}$ direction $\left(p_{1} \neq 0, p_{2}=0, p_{3}=0\right)$ obey

$$
\left(p_{0}+p_{1} \gamma^{t} \gamma^{1}\right) \Phi(p)=\left(\begin{array}{cccc}
p_{0}+p_{1} & 0 & 0 & 0 \\
0 & p_{0}-p_{1} & 0 & 0 \\
0 & 0 & p_{0}+p_{1} & 0 \\
0 & 0 & 0 & p_{0}-p_{1}
\end{array}\right)\left(\begin{array}{l}
\Phi_{1}^{+}(p) \\
\Phi_{2}^{-}(p) \\
\Phi_{3}^{+}(p) \\
\Phi_{4}^{-}(p)
\end{array}\right) .=0 .
$$

The equation in (5.43) describes that that (anti-)particles can propagate along only the left or right direction as follow. An anti-particle $\Phi^{+}(p)\left(\gamma^{t} \gamma^{1} \Phi^{+}(p)=\Phi^{+}(p)\right)$ can not propagate in the right direction parallel to $x^{1}$ axis $\left(x^{1}>0\right)$. On the other hand, the anti-particle $\Phi^{-}(p)\left(\gamma^{t} \gamma^{1} \Phi^{-}(p)=-\Phi^{-}(p)\right)$ is not able to propagate in the left direction $\left(x^{1}<0\right)$. Therefore if we define a locally excited state by acting a component of $\psi$ or $\bar{\psi}$ on the ground state, their reduced density matrices depend on the representation of gamma matrices as in (5.8) and (5.9). For example, we choose the basis which diagonalizes $\gamma^{t} \gamma^{1}$ as in (5.42) and $\psi_{1}$ acts on the ground state. This local operator creates an anti-particle and it propagates with time. At the late time $(t \gg l)$, it is necessarily included in the region $\mathrm{A}$ or B. As explained above, it is not able to propagate in the right direction parallel to $x^{1}$ axis. Therefore it is included in $A(B)$ with probability which is bigger than $\frac{1}{2}$ (less than $\left.\frac{1}{2}\right)$. The reduced density matrix in (5.8) is given by

$$
\rho_{A}=\frac{1}{4}\left(\begin{array}{ll}
3 & 0 \\
0 & 1
\end{array}\right) .
$$

The result in (5.44) describes that the antiparticle which created by $\psi_{1}$ are included in A (B) with probability $\frac{3}{4}\left(\frac{1}{4}\right)^{6} \cdot{ }^{7}$ If we choose a basis in which $\left(\gamma^{t} \gamma^{1}\right)_{11}$ is given by $0, \Delta S_{A}^{(n)}$ is given by $\log 2$.

\footnotetext{
${ }^{6}$ If we act $\psi_{1}+\psi_{2}$ on the ground state, the late time value of $\Delta S_{A}^{(2)}$ is given by $\log 2$ as we expected

${ }^{7}$ If you choose the bases which diagonalize $\gamma^{t} \gamma^{1}$ in $2 \mathrm{~d}$ massless free fermionic field theory, a component of $\psi(\bar{\psi})$ is able to propagate in the only left or right direction parallel to $x^{1}$. Therefore $\Delta S_{A}^{(n)}$ vanish for the locally excited states generated by acting a component of $\psi(\bar{\psi})$ on the ground state.
} 


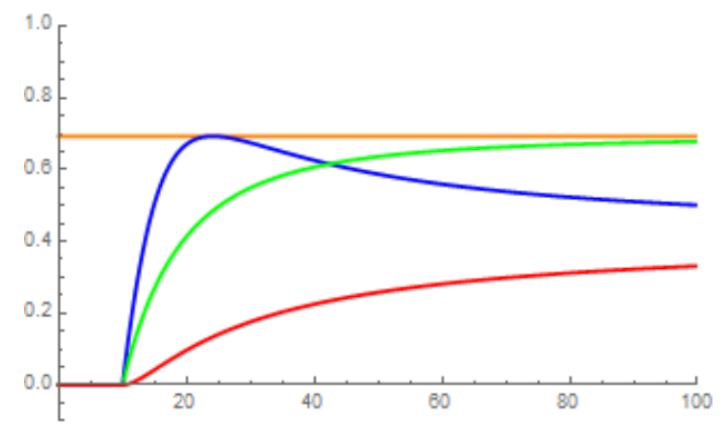

Figure 8. Plots of $\Delta S_{A}^{(3)}$ for $\mathcal{N} \psi_{a}|0\rangle$ changing the value of $\left(\gamma^{t} \gamma^{1}\right)_{a a}$. Here $l=10$. The vertical and horizontal lines correspond to $\Delta S_{A}^{(n)}$ and time $t$ respectively. The blue line, green line and red line correspond to $\Delta S_{A}^{(3)}$ for $\left(\gamma^{t} \gamma^{1}\right)_{a a}=-1,\left(\gamma^{t} \gamma^{1}\right)_{a a}=0$ and $\left(\gamma^{t} \gamma^{1}\right)_{a a}=1$ respectively. The orange line corresponds to $\log 2$.

\subsubsection{Spin dependence of time evolution}

Here we discuss the spin dependence of the time evolution of $\Delta S_{A}^{(n)}$. A locally excited state of a single fermionic operator is created as in (5.1) and the entropy $\Delta S_{A}^{(n)}$ evolves as in (5.4). An interesting feature that didn't show up in the previous works on the bosonic quenches $[23-25,30]$ is that $\Delta S_{A}^{(n)}$ is not a monotonic function of time and it can decrease for some period of time. In figure 8, we show the time evolution of $\Delta S_{A}^{(3)}$ for the various values of $\left(\gamma^{t} \gamma^{1}\right)_{a a}$. As one can see, $\Delta S_{A}^{(3)}$ monotonically increases for $\left(\gamma^{t} \gamma^{1}\right)_{a a} \geq 0$ while it increases to reach the maximum value $\log 2$ at a finite time and then decreases to an asymptotic value for $\left(\gamma^{t} \gamma^{1}\right)_{a a}<0$.

One can interpret it as follows. After the local quench, the fermion propagates in all directions at the speed of light. However, the probability is not uniformly distributed. In our convention, the states $\psi_{a}$ which have $\left(\gamma^{t} \gamma^{1}\right)_{a a}>0$ are more likely to propagate in the negative $x_{1}$ direction and those with $\left(\gamma^{t} \gamma^{1}\right)_{a a}<0$ are more likely to propagate in the positive $x_{1}$ direction. If $\left(\gamma^{t} \gamma^{1}\right)_{a a}>0$ the major part of the wavefunction stays in the subsystem $B$ and the probability of finding an anti-particle in the subsystem $A$ is always less than a half. Therefore, any wave coming into $A$ contributes positively to the entropy. On the other hand, if $\left(\gamma^{t} \gamma^{1}\right)_{a a}<0$, the major part of the wavefunction comes into $A$. After $t=l$, the excitation wave created in $B$ starts to enter the subsystem $A$ and so the entropy starts to increase. At a critical time $t=t^{*}$, the probability of finding an anti-particle in $A$ becomes equal to that in $B$ and the entropy reaches its maximum value $\log 2$. From the structure of the entropy, one cannot share more information between $A$ and $B$ than this. The excitation wave keeps entering $A$ after $t=t^{*}$, but this effectively reduces the sharing information between the subsystems. Therefore, $\Delta S_{A}^{(n)}$ starts to decrease after $t=t^{*}$. The critical time $t^{*}$ is given by

$$
t^{*}=-\frac{\left(\sqrt{\left(\left(\gamma^{t} \gamma^{1}\right)_{a a}\right)^{2}+1}+1\right) l}{\left(\gamma^{t} \gamma^{1}\right)_{a a}} .
$$

This value does not depend on the replica number $n$. 


\section{Quasi-particle interpretation}

In [23-25], we have interpreted the late time value of $\Delta S_{A}^{(n)}$ in free massless scalar field theories in terms of entanglement between quasi-particles.

In free fermionic field theory, it is expected that the late time values of $\Delta S_{A}^{(n)}$ can be interpreted in terms of entanglement between quasi-particles. Therefore we decompose local operators into left moving modes and right moving modes,

$$
\begin{aligned}
\psi_{a} & =\psi_{a}^{L \dagger}+\psi_{a}^{R \dagger}+\phi_{a}^{L}+\phi_{a}^{R}, \\
\psi_{a}^{\dagger} & =\psi_{a}^{L}+\psi_{a}^{R}+\phi_{a}^{L \dagger}+\phi_{a}^{R \dagger}, \\
\bar{\psi}_{a} & =\bar{\psi}_{a}^{L \dagger}+\bar{\psi}_{a}^{R \dagger}+i\left(\psi^{L} \gamma^{t}\right)_{a}+i\left(\psi^{R} \gamma^{t}\right)_{a},
\end{aligned}
$$

where $\bar{\psi}^{K \dagger}$ is defined by

$$
\begin{aligned}
& \bar{\psi}_{a}^{L \dagger} \equiv i\left(\phi^{L \dagger} \gamma^{t}\right)_{a} \\
& \bar{\psi}_{a}^{R \dagger} \equiv i\left(\phi^{R \dagger} \gamma^{t}\right)_{a}
\end{aligned}
$$

The left moving mode and right moving mode of the anti-particle and particle are defined as follows. We decompose $\psi, \psi^{\dagger}$ into the momentum modes. Their right moving modes (left moving modes) are defined by the sum of the momentum modes whose $\frac{p_{0}}{p_{1}}$ is positive ( $\frac{p_{0}}{p_{1}}$ is negative). As we explained in the previous section, the number of momentum modes which the right moving modes (left moving modes) depends on the choice of spin's direction. Therefore instead of ordinary anti-commutation relationships $\left(\left\{\Phi_{a}^{K}, \Phi_{b}^{K^{\prime} \dagger}\right\}=\right.$ $\left.\delta_{K K^{\prime}} \delta_{a b}\right)$, we impose the following exotic anti-commutation relationship on the particle and anti-particle,

$$
\begin{aligned}
\left\{\phi_{a}^{R}, \phi_{b}^{R \dagger}\right\} & =\left(\delta_{a b}-\frac{1}{2}\left(\gamma^{t} \gamma^{1}\right)_{a b}\right), \\
\left\{\phi_{a}^{L}, \phi_{b}^{L \dagger}\right\} & =\left(\delta_{a b}+\frac{1}{2}\left(\gamma^{t} \gamma^{1}\right)_{a b}\right), \\
\left\{\psi_{a}^{R \dagger}, \psi_{b}^{R}\right\} & =\left(\delta_{a b}-\frac{1}{2}\left(\gamma^{t} \gamma^{1}\right)_{a b}\right), \\
\left\{\psi_{a}^{L \dagger}, \psi_{b}^{L}\right\} & =\left(\delta_{a b}+\frac{1}{2}\left(\gamma^{t} \gamma^{1}\right)_{a b}\right), \\
\left\{\bar{\psi}_{a}^{R}, \bar{\psi}_{b}^{R \dagger}\right\} & =\left(\delta_{a b}-\frac{1}{2}\left(\gamma^{1} \gamma^{t}\right)_{a b}\right), \\
\left\{\bar{\psi}_{a}^{L}, \bar{\psi}_{b}^{L \dagger}\right\} & =\left(\delta_{a b}+\frac{1}{2}\left(\gamma^{1} \gamma^{t}\right)_{a b}\right) .
\end{aligned}
$$

The vacuum $|0\rangle=|0\rangle_{L} \otimes|0\rangle_{R}$ is defined by

$$
\phi_{a}^{K}|0\rangle=\psi_{a}^{K}|0\rangle=\bar{\psi}_{a}^{K}|0\rangle=0,
$$

where $K=L, R$. The number of momentum modes which the left and right moving modes have depends on the choice of $\gamma^{t} \gamma^{1}$ (the choice of the spin's direction). Therefore, it is 
reasonable that the anti-commutation for them is given by that in (6.3). ${ }^{8}$ We claim that the exotic anti-commutation relationship in (6.3) can be applied to $\Delta S_{A}^{(n)}$ in 4 dimensional free massless fermionic field theory. However in $d(\neq 4)$ dimensional spacetime, that in $(6.3)$ should be deformed. For example, in 2 dimensional spacetime the reduced density matrix for the state excited by a local operator constructed of chiral and anti-chiral operators should become

$$
\rho_{A}=\frac{1}{2}\left(\begin{array}{cc}
1+\left(\gamma^{t} \gamma^{1}\right)_{a a} & 0 \\
0 & 1-\left(\gamma^{t} \gamma^{1}\right)_{a a}
\end{array}\right) .
$$

Therefore the anti-commutation relation in (6.3) should change to another one.

This way, we can construct reduced density matrices which agree with those which are obtained by the replica trick as we will see later. In following subsections we will compute them under this decomposition in various examples in 4 dimensional free massless fermionic field theory.

\subsection{Various examples}

Here we compute reduced density matrices for various locally excited states under the quasi-particle interpretation.

\subsection{1 $\rho_{A}$ for $\psi_{a}$}

A locally excited states is given by

$$
|\Psi\rangle=\mathcal{N} \psi_{a}|0\rangle
$$

which is variant under the $\operatorname{SL}(2, \mathbf{C})$ transformation. Under the decomposition in (6.1), this state is given by

$$
|\Psi\rangle=\frac{1}{\sqrt{2}}\left[\psi_{a}^{L \dagger}|0\rangle_{L} \otimes|0\rangle_{R}+|0\rangle_{L} \otimes \psi_{a}^{R \dagger}|0\rangle_{R}\right],
$$

after the normalization constant $\mathcal{N}$ is determined.

Normalized orthogonal left and right moving states are given by

$$
\begin{aligned}
& |0\rangle_{L}, \\
& |0\rangle_{R}, \\
& \left|\psi_{a}^{L}\right\rangle_{L}=\mathcal{N}^{L} \psi_{a}^{L \dagger}|0\rangle_{L}, \\
& \left|\psi_{a}^{R}\right\rangle_{R}=\mathcal{N}^{R} \psi_{a}^{R \dagger}|0\rangle_{R},
\end{aligned}
$$

where $\left(\mathcal{N}^{L}\right)^{2}$ and $\left(\mathcal{N}^{R}\right)^{2}$ are given by

$$
\begin{aligned}
\left(\mathcal{N}^{L}\right)^{2} & =\frac{1}{1+\frac{\left(\gamma^{t} \gamma^{1}\right)_{a a}}{2}} \\
\left(\mathcal{N}^{R}\right)^{2} & =\frac{1}{1-\frac{\left(\gamma^{t} \gamma^{1}\right)_{a a}}{2}} .
\end{aligned}
$$

\footnotetext{
${ }^{8}$ However we find this exotic anti-commutation relationship heuristically.
} 
A reduced density matrix $\rho_{A}$ is defined by $\operatorname{tr}_{L} \rho$. It is given by

$$
\rho_{A}=\frac{1}{4}\left[\left(2+\left(\gamma^{t} \gamma^{1}\right)_{a a}\right)|0\rangle_{R}\left\langle\left. 0\right|_{R}+\left(2-\left(\gamma^{t} \gamma^{1}\right)_{a a}\right) \mid \psi_{a}^{R}\right\rangle_{R}\left\langle\left.\psi_{a}^{R}\right|_{R}\right]\right.
$$

which agrees with the one obtained by the replica trick. When $\left(\gamma^{t} \gamma^{1}\right)_{a a}$ vanishes, $\Delta S_{A}^{(n)}$ are given by $\log 2$. In the $n \rightarrow 1$ limit, it is given by entanglement entropy for maximally entangled state (EPR state). If we choose gamma matrices as in (5.42), the anti-particles created by $\psi_{1}$ is not able to propagate in the right direction parallel to $x^{1}$ axis. Therefore probability with which it is finally included in $B$ is larger than the one with which it is included in $A$ at the late time. The reduced density matrix in (6.10) agrees with our intuitive expectation.

\subsection{2 $\rho_{A}$ for $\bar{\psi} \psi$}

A locally excited states is given by

$$
|\Psi\rangle=\mathcal{N} \bar{\psi} \psi|0\rangle
$$

which is invariant under the $\mathrm{SL}(2, \mathbf{C})$ transformation.

By using an unitary transformation, $\gamma^{t} \gamma^{1}$ can be diagonalized. After diagonalize it, the reduced density matrix for this state can be computed easily. Here it is given by that in (5.42).

Under the decomposition in (6.1), the state in (6.11) is given by

$$
|\Psi\rangle=\frac{1}{4} \sum_{a}\left[\bar{\psi}_{a}^{L \dagger} \psi_{a}^{L \dagger}+\bar{\psi}_{a}^{L \dagger} \psi_{a}^{R \dagger}+\bar{\psi}_{a}^{R \dagger} \psi_{a}^{L \dagger}+\bar{\psi}_{a}^{R \dagger} \psi_{a}^{R \dagger}\right]|0\rangle_{L} \otimes|0\rangle_{R},
$$

after the normalization constant $\mathcal{N}$ is determined.

Normalized orthogonal states are given by

$$
\begin{aligned}
& |0\rangle_{L}, \\
& |0\rangle_{R}, \\
& \left|\bar{\psi}^{L} \psi^{L}\right\rangle_{L}=\mathcal{N}^{L L} \sum_{a} \bar{\psi}_{a}^{L \dagger} \psi_{a}^{L \dagger}|0\rangle_{L}, \\
& \left|\bar{\psi}^{R} \psi^{R}\right\rangle_{R}=\mathcal{N}^{R R} \sum_{a} \bar{\psi}_{a}^{R \dagger} \psi_{a}^{R \dagger}|0\rangle_{R}, \\
& \left|\psi_{a}^{L}\right\rangle=\tilde{\mathcal{N}}_{a}^{L} \psi_{a}^{L \dagger}|0\rangle_{L}, \\
& \left|\psi_{a}^{R}\right\rangle=\tilde{\mathcal{N}}_{a}^{R} \psi_{a}^{R \dagger}|0\rangle_{R}, \\
& \left|\bar{\psi}_{a}^{L}\right\rangle=\overline{\mathcal{N}}_{a}^{L} \psi_{a}^{L \dagger}|0\rangle_{L}, \\
& \left|\bar{\psi}_{a}^{R}\right\rangle=\overline{\mathcal{N}}_{a}^{R} \psi_{a}^{R \dagger}|0\rangle_{R},
\end{aligned}
$$

where normalization factors are given by

$$
\begin{aligned}
& \left(\mathcal{N}^{L L}\right)^{2}=\left(\mathcal{N}^{R R}\right)^{2}=\frac{1}{3}, \\
& \left(\tilde{\mathcal{N}}_{1}^{R}\right)^{2}=\left(\tilde{\mathcal{N}}_{3}^{R}\right)^{2}=\left(\tilde{\mathcal{N}}_{2}^{L}\right)^{2}=\left(\tilde{\mathcal{N}}_{4}^{L}\right)^{2}=\left(\overline{\mathcal{N}}_{2}^{R}\right)^{2}=\left(\overline{\mathcal{N}}_{4}^{R}\right)^{2}=\left(\overline{\mathcal{N}}_{1}^{L}\right)^{2}=\left(\overline{\mathcal{N}}_{3}^{L}\right)^{2}=2, \\
& \left(\tilde{\mathcal{N}}_{2}^{R}\right)^{2}=\left(\tilde{\mathcal{N}}_{4}^{R}\right)^{2}=\left(\tilde{\mathcal{N}}_{1}^{L}\right)^{2}=\left(\tilde{\mathcal{N}}_{3}^{L}\right)^{2}=\left(\overline{\mathcal{N}}_{1}^{R}\right)^{2}=\left(\overline{\mathcal{N}}_{3}^{R}\right)^{2}=\left(\overline{\mathcal{N}}_{2}^{L}\right)^{2}=\left(\overline{\mathcal{N}}_{4}^{L}\right)^{2}=\frac{2}{3} .
\end{aligned}
$$


A reduce density matrix is given by

$$
\begin{aligned}
\rho_{A}=\operatorname{tr}_{L} \rho= & \frac{12}{64}|0\rangle_{R}\left\langle\left. 0\right|_{R}+\sum_{i} \frac{\mathcal{M}_{i}}{64} \mid \psi_{i}^{R}\right\rangle_{R}\left\langle\left.\psi_{i}^{R}\right|_{R}\right. \\
& +\sum_{i} \frac{\overline{\mathcal{M}}_{i}}{64}\left|\bar{\psi}_{i}^{R}\right\rangle_{R}\left\langle\left.\bar{\psi}_{i}^{R}\right|_{R}+\frac{12}{64} \mid \bar{\psi}^{R} \psi^{R}\right\rangle_{R}\left\langle\left.\bar{\psi}^{R} \psi^{R}\right|_{R},\right.
\end{aligned}
$$

where $\mathcal{M}_{i}$ and $\overline{\mathcal{M}}_{i}$ are given by

$$
\begin{aligned}
& \mathcal{M}_{1}=\mathcal{M}_{3}=\overline{\mathcal{M}}_{2}=\overline{\mathcal{M}}_{4}=1, \\
& \mathcal{M}_{2}=\mathcal{M}_{4}=\overline{\mathcal{M}}_{1}=\overline{\mathcal{M}}_{3}=9 .
\end{aligned}
$$

This reduced density matrix agrees with the one obtained by the replica trick.

\subsection{3 $\rho_{A}$ for $\psi^{\dagger} \psi$}

A locally excited states is given by

$$
|\Psi\rangle=\mathcal{N} \psi^{\dagger} \psi|0\rangle
$$

which is variant under the $\operatorname{SL}(2, \mathbf{C})$ transformation. However $\Delta S_{A}^{(n)}$ for (6.17) is invariant in the replica trick. Therefore we diagonalize $\gamma^{t} \gamma^{1}$ as in (5.42). After diagonalizing it, $\rho_{A}$ is given by

$$
\begin{aligned}
\rho_{A}=\operatorname{tr}_{R} \rho= & \frac{20}{64}\left|\phi^{R} \psi^{R}\right\rangle_{R}\left\langle\left.\phi^{R} \psi^{R}\right|_{R}+\frac{20}{64} \mid 0\right\rangle_{R}\left\langle\left. 0\right|_{R}\right. \\
& +\frac{3}{64} \sum_{i}\left|\phi_{i}^{R}\right\rangle_{R}\left\langle\left.\phi_{i}^{R}\right|_{R}+\frac{3}{64} \sum_{i} \mid \psi_{i}^{R}\right\rangle_{R}\left\langle\left.\psi_{i}^{R}\right|_{R} .\right.
\end{aligned}
$$

\section{Conclusions and discussions}

In this paper we have studied the dynamics of quantum entanglement. We considered the 4 dimensional free massless fermionic field theory. Locally excited states are generated by being acted on the ground state by various local operators. We defined the excesses of (Rényi) entanglement entropies $\Delta S_{A}^{(n)}$ by subtracting (Rényi) entanglement entropies for the ground state from those for locally excited states. $\Delta S_{A}^{(n)}$ are finite quantities. We found that $\Delta S_{A}^{(n)}$ vanish if $t \leq l$. If $t>l, \Delta S_{A}^{(n)}$ monotonically increase and finally approach constants for the states in (5.10) and (5.28) as in scalar field theory [23-25]. For the state in (5.1), $\Delta S_{A}^{(n)}$ does not necessarily monotonically increase in $t>l$. Depending on the representation of $\left(\gamma^{t} \gamma^{1}\right)_{a a}$, it can increase monotonically before $t=t^{*}$ and maximize at $t=t^{*}$ and decrease and approache a constant after that.

We found that if the locally excited state is given by $\mathcal{N} \psi_{a}|0\rangle, \mathcal{N} \bar{\psi}_{a}|0\rangle$, components of its reduced density matrices include $\gamma^{t} \gamma^{1}$. Therefore they depend on the direction of spin. A component of $\psi$ or $\bar{\psi}$ creates an anti-particle or particle. Their propagation depends on the direction of spin. Therefore components of reduced density matrices for those locally excited states depend on the direction of spin. We also generated locally excited 
states $\mathcal{N} \psi^{\dagger} \psi|0\rangle$ and $\mathcal{N} \bar{\psi} \psi|0\rangle$ and investigated the time evolution of $\Delta S_{A}^{(n)}$ for them. We found that $\Delta S_{A}^{(n)}$ are invariant under the lorentz transformation although $\mathcal{N} \psi^{\dagger} \psi|0\rangle$ is not invariant under the lorentz transformation.

We found that the time evolution and the late time values of $\Delta S_{A}^{(n)}$ can be interpreted in terms of quasi-particles. However in the fermionic field theory, the number of modes which propagate in the left $\left(x^{1}<0\right)$ or the right $\left(x^{1}>0\right)$ direction depend on the choice of the spin's direction. Therefore we need to impose exotic anti-commutation relation in (6.3) on quasi-particles. In the 4 dimensional free massless fermionic field theory, the results by the replica trick can be interpreted as the quantum entanglement between quasi-particles if the relationship in $(6.3)$ is applied to them. In the $d(\neq 4)$ dimensional free fermionic field theory, anti-commutation relationship should be changed.

We would like to list a few of future problems:

- It is interesting to study charged (Rényi) entanglement entropies for locally excited stats and holographic field theory.

- It is important to study how we should deform the relationship in $(6.3)$ in $d(\neq 4)$ dimensional free massless fermionic field theory.

- It is interesting to study the time evolution of $\Delta S_{A}^{(n)}$ in non-relativistic field theory.

- It would be expected that gamma matrices included in reduced density matrices is related to the modular Hamiltonian. It is interesting to clarify the relationship between the spin dependence of the reduced matrices and the entanglement Hamiltonian.

\section{Acknowledgments}

MN thanks Tadashi Takayanagi and Masaharu Tanabashi for useful discussions and advices. We thank Mitsutoshi Fujita for collaboration in the early stage of this work. We also thank Tatsuma Nishioka, Daniel Grumiller, Max Riegler and Pawel Caputa for useful discussions.

Open Access. This article is distributed under the terms of the Creative Commons Attribution License (CC-BY 4.0), which permits any use, distribution and reproduction in any medium, provided the original author(s) and source are credited.

\section{References}

[1] C. Holzhey, F. Larsen and F. Wilczek, Geometric and renormalized entropy in conformal field theory, Nucl. Phys. B 424 (1994) 443 [hep-th/9403108] [INSPIRE].

[2] G. Vidal, J.I. Latorre, E. Rico and A. Kitaev, Entanglement in quantum critical phenomena, Phys. Rev. Lett. 90 (2003) 227902 [quant-ph/0211074] [INSPIRE].

[3] J.I. Latorre, E. Rico and G. Vidal, Ground state entanglement in quantum spin chains, Quant. Inf. Comput. 4 (2004) 48 [quant-ph/0304098] [INSPIRE].

[4] P. Calabrese and J.L. Cardy, Entanglement entropy and quantum field theory, J. Stat. Mech. 0406 (2004) P06002 [hep-th/0405152] [INSPIRE]. 
[5] P. Calabrese, and A. Lefevre, Entanglement spectrum in one-dimensional systems, Phys. Rev. A 78 (2008) 032329 [arXiv:0806.3059].

[6] A. Kitaev and J. Preskill, Topological entanglement entropy, Phys. Rev. Lett. 96 (2006) 110404 [hep-th/0510092] [inSPIRE].

[7] M. Levin and X.G. Wen, Detecting topological order in a ground state wave function, Phys. Rev. Lett. 96 (2006) 110405 [cond-mat/0510613].

[8] S. Ryu and T. Takayanagi, Holographic derivation of entanglement entropy from AdS/CFT, Phys. Rev. Lett. 96 (2006) 181602 [hep-th/0603001] [INSPIRE].

[9] S. Ryu and T. Takayanagi, Aspects of holographic entanglement entropy, JHEP 08 (2006) 045 [hep-th/0605073] [inSPIRE].

[10] V.E. Hubeny, M. Rangamani and T. Takayanagi, A covariant holographic entanglement entropy proposal, JHEP 07 (2007) 062 [arXiv:0705.0016] [INSPIRE].

[11] T. Nishioka, S. Ryu and T. Takayanagi, Holographic entanglement entropy: an overview, J. Phys. A 42 (2009) 504008 [arXiv: 0905.0932] [INSPIRE].

[12] T. Takayanagi, Entanglement entropy from a holographic viewpoint, Class. Quant. Grav. 29 (2012) 153001 [arXiv:1204.2450] [inSPIRE].

[13] M. Van Raamsdonk, Building up spacetime with quantum entanglement, Gen. Rel. Grav. 42 (2010) 2323 [Int. J. Mod. Phys. D 19 (2010) 2429] [arXiv:1005.3035] [InSPIRE].

[14] M.B. Hastings, An area law for one dimensional quantum systems, J. Stat. Mech. (2007) P08024 [arXiv:0705.2024].

[15] Y. Chen and G. Vidal, Entanglement contour, J. Stat. Mech. (2014) P10011 [arXiv:1406.1471].

[16] M. Nozaki, T. Numasawa and T. Takayanagi, Holographic local quenches and entanglement density, JHEP 05 (2013) 080 [arXiv: 1302.5703] [INSPIRE].

[17] J. Eisert, M. Friesdorf and C. Gogolin, Quantum many-body systems out of equilibrium, Nature Phys. 11 (2015) 124.

[18] A. Lamacraft and J. Moore, Ultracold bosonic and fermionic gases, volume 5, Contemporary Concepts of Condensed Matter Science, Elsevier, Oxford U.K. (2012).

[19] P. Calabrese and J.L. Cardy, Evolution of entanglement entropy in one-dimensional systems, J. Stat. Mech. 0504 (2005) P04010 [cond-mat/0503393] [INSPIRE].

[20] P. Calabrese and J. Cardy, Entanglement entropy and conformal field theory, J. Phys. A 42 (2009) 504005 [arXiv:0905.4013] [INSPIRE].

[21] V. Eisler, F. Igloi and I. Peschel, Evolution of entanglement after a local quench, J. Stat. Mech. (2007) P06005 [cond-mat/0703379].

[22] F.C. Alcaraz, M.I. Berganza and G. Sierra, Entanglement of low-energy excitations in conformal field theory, Phys. Rev. Lett. 106 (2011) 201601 [arXiv:1101.2881] [INSPIRE].

[23] M. Nozaki, T. Numasawa and T. Takayanagi, Quantum entanglement of local operators in conformal field theories, Phys. Rev. Lett. 112 (2014) 111602 [arXiv:1401.0539] [INSPIRE].

[24] M. Nozaki, Notes on quantum entanglement of local operators, JHEP 10 (2014) 147 [arXiv: 1405.5875] [INSPIRE]. 
[25] P. Caputa, M. Nozaki and T. Takayanagi, Entanglement of local operators in large- $N$ conformal field theories, PTEP 2014 (2014) 093B06 [arXiv: 1405.5946] [INSPIRE].

[26] S. He, T. Numasawa, T. Takayanagi and K. Watanabe, Quantum dimension as entanglement entropy in two dimensional conformal field theories, Phys. Rev. D 90 (2014) 041701 [arXiv: 1403.0702] [INSPIRE].

[27] B. Chen, W.-Z. Guo, S. He and J.-q. Wu, Entanglement entropy for descendent local operators in $2 D$ CFTs, JHEP 10 (2015) 173 [arXiv:1507.01157] [INSPIRE].

[28] W.-Z. Guo and S. He, Rényi entropy of locally excited states with thermal and boundary effect in 2D CFTs, JHEP 04 (2015) 099 [arXiv: 1501.00757] [INSPIRE].

[29] P. Caputa and A. Veliz-Osorio, Entanglement constant for conformal families, Phys. Rev. D 92 (2015) 065010 [arXiv: 1507.00582] [INSPIRE].

[30] P. Caputa, J. Simón, A. Stikonas and T. Takayanagi, Quantum entanglement of localized excited states at finite temperature, JHEP 01 (2015) 102 [arXiv:1410.2287] [INSPIRE].

[31] C.T. Asplund, A. Bernamonti, F. Galli and T. Hartman, Holographic entanglement entropy from $2 D$ CFT: heavy states and local quenches, JHEP 02 (2015) 171 [arXiv:1410.1392] [INSPIRE].

[32] A. Belin, L.-Y. Hung, A. Maloney, S. Matsuura, R.C. Myers and T. Sierens, Holographic charged Rényi entropies, JHEP 12 (2013) 059 [arXiv:1310.4180] [INSPIRE].

[33] J. Bhattacharya, M. Nozaki, T. Takayanagi and T. Ugajin, Thermodynamical property of entanglement entropy for excited states, Phys. Rev. Lett. 110 (2013) 091602 [arXiv: 1212.1164] [INSPIRE].

[34] D.D. Blanco, H. Casini, L.-Y. Hung and R.C. Myers, Relative entropy and holography, JHEP 08 (2013) 060 [arXiv: 1305.3182] [INSPIRE].

[35] G. Wong, I. Klich, L.A. Pando Zayas and D. Vaman, Entanglement temperature and entanglement entropy of excited states, JHEP 12 (2013) 020 [arXiv:1305.3291] [INSPIRE].

[36] M.E.X. Guimaraes and B. Linet, Scalar Green's functions in an Euclidean space with a conical-type line singularity, Commun. Math. Phys. 165 (1994) 297 [INSPIRE].

[37] M. A. Metlitski, C.A. Fuertes and S. Sachdev, Entanglement Entropy in the $O(N)$ model, Phys. Rev. B 80 (2009) 115122 [arXiv:0904.4477].

[38] B. Linet, Euclidean spinor Green's functions in the space-time of a straight cosmic string, J. Math. Phys. 36 (1995) 3694 [gr-qc/9412050] [INSPIRE].

[39] H. Casini, M. Huerta and R.C. Myers, Towards a derivation of holographic entanglement entropy, JHEP 05 (2011) 036 [arXiv: 1102.0440] [INSPIRE].

[40] H. Casini and M. Huerta, Entanglement entropy in free quantum field theory, J. Phys. A 42 (2009) 504007 [arXiv:0905.2562] [INSPIRE]. 